\title{
Sulforaphane prevents and reverses allergic airways disease in mice via anti-inflammatory, antioxidant and epigenetic mechanisms
}

\section{Simon Royce}

Monash University

Paul Licciardi

Murdoch Children's Research Institute https://orcid.org/0000-0001-6086-6285

Raymond Beh

Monash University https://orcid.org/0000-0001-9801-1549

Jane Bourke

Monash University https://orcid.org/0000-0001-7314-9234

Mark Cooper

Monash University https://orcid.org/0000-0002-5953-642X

\section{Chantal Donovan}

Hunter Medical Research Institute, University of Newcastle

\section{Andrew Hung}

RMIT University

\section{Ishant Khurana}

Epigenetics in Human Health and Disease Laboratory, Department of Diabetes, Central Clinical School, Monash University, Melbourne

Julia Liang

Monash University https://orcid.org/0000-0002-3031-3169

\section{Scott Maxwell}

Monash University, Alfred Centre https://orcid.org/0000-0002-7801-0714

\section{Nadia Mazarakis}

Monash University https://orcid.org/0000-0001-7681-2775

\section{Eleni Pitsillou}

Monash University

\section{Kenneth Snibson}

University of Melbourne

\section{Mark Tobin}

Australian Synchrotron https://orcid.org/0000-0003-1862-0649

\section{Katherine Ververis}

Monash University 


\section{Jitraporn Vongsvivut}

Australian Synchrotron

\section{Mark Ziemann}

Deakin University

\section{Chrishan Samuel}

Monash University https://orcid.org/0000-0003-0295-4214

\section{Mimi Tang}

Royal Children's Hospital https://orcid.org/0000-0002-3839-5293

\section{Sam El-Osta}

Monash University https://orcid.org/0000-0001-7968-7375

Tom Karagiannis ( $\nabla$ tom.karagiannis@monash.edu )

Monash University https://orcid.org/0000-0002-9967-1546

\section{Article}

Keywords: sulforaphane, allergic airways disease, L-sulforaphane, epigenetic regulatory mechanisms

Posted Date: August 23rd, 2021

DOl: https://doi.org/10.21203/rs.3.rs-787983/v1

License: (c) (1) This work is licensed under a Creative Commons Attribution 4.0 International License. Read Full License 
Sulforaphane prevents and reverses allergic airways disease in mice via anti-inflammatory, antioxidant and epigenetic mechanisms

3 Simon G. Royce ${ }^{1,2,3,4}$, Paul V. Licciardi ${ }^{4,5}$, Raymond C. Beh ${ }^{1,2}$, Jane E. Bourke ${ }^{3}$, Mark E.

4 Cooper $^{6,7}$, Chantal Donovan ${ }^{8,9,10}$, Andrew Hung ${ }^{11}$, Ishant Khurana ${ }^{12}$, Julia Liang ${ }^{1,11}$, Scott

5 Maxwell $^{12}$, Nadia Mazarakis ${ }^{1,4,5,13}$, Eleni Pitsillou ${ }^{1,11}$, Kenneth J. Snibson ${ }^{13}$, Mark J. Tobin ${ }^{14}$,

6 Katherine Ververis ${ }^{1,2}$, Jitraporn Vongsvivut ${ }^{14}$, Mark Ziemann ${ }^{12,15}$, Chrishan S. Samuel ${ }^{16}$, Mimi

$7 \quad$ L.K Tang ${ }^{5,17,18}$, Assam El-Osta ${ }^{12}$ \& Tom C. Karagiannis ${ }^{1,2, *}$

81 Epigenomic Medicine Laboratory, Department of Diabetes, Central Clinical School, Monash

University, Alfred Centre, 99 Commercial Road, Melbourne, VIC, 3004, Australia

2 Department of Clinical Pathology, University of Melbourne, Parkville, VIC, 3010, Australia

3 Monash University, Department of Pharmacology, Biomedicine Discovery Institute, Clayton, VIC, 3800, Australia

4 Infection and Immunity, Murdoch Children's Research Institute, Melbourne, VIC, 3052,

Australia

5 Department of Paediatrics, The University of Melbourne, Melbourne, VIC, 3010, Australia

6 Department of Diabetes, Central Clinical School, Monash University, Alfred Centre, 99

Commercial Road, Melbourne, VIC, 3004, Australia

7 Department of Endocrinology and Diabetes, The Alfred Hospital, Melbourne, VIC, 3004, Australia

8 Priority Research Centre for Healthy Lungs, Hunter Medical Research Institute, University of Newcastle, Newcastle, NSW, 2305, Australia

9 Centre for Inflammation, Centenary Institute, Camperdown, NSW, 2050, Australia

10 University of Technology Sydney, School of Life Sciences, Faculty of Science, Sydney, NSW, 2007, Australia

11 School of Science, STEM College, RMIT University, VIC, 3001, Australia

12 Epigenetics in Human Health and Disease Laboratory, Department of Diabetes, Central Clinical School, Monash University, Alfred Centre, 99 Commercial Road, Melbourne, VIC, 3004, Australia

13 Faculty of Veterinary and Agricultural Sciences, University of Melbourne, Parkville, VIC, 3010, Australia

14 ANSTO-Australian Synchrotron, Clayton, VIC, 3168, Australia

15 School of Life and Environmental Sciences, Deakin University, Waurn Ponds, VIC, 3216,

Australia

16 Cardiovascular Disease Program, Monash Biomedicine Discovery Institute and Department of Pharmacology, Monash University, Clayton, VIC, 3800, Australia

17 Population Allergy Group, Murdoch Children's Research Institute, Parkville, VIC, 3052, Australia

18 Department of Allergy and Immunology, Royal Children's Hospital, Parkville, VIC, 3052,

Australia

Short title: Sulforaphane attenuates allergic airways disease

*Author for correspondence:

Tom Karagiannis, PhD

Head Epigenomic Medicine Program

Department of Diabetes, Central Clinical School, Monash University

Melbourne, VIC 3004, Australia

46 Email: tom.karagiannis@ monash.edu ; Phone number: +61 400857906 


\section{Abstract}

2 Sulforaphane has been investigated in human pathologies and preclinical models of airway

3 diseases. To provide further mechanistic insights, we explored L-sulforaphane (LSF) in the

4 ovalbumin (OVA)-induced chronic allergic airways murine model, with key hallmarks of

5 asthma. Histological analysis indicated that LSF prevented or reversed OVA-induced epithelial

6 thickening, collagen deposition, goblet cell metaplasia, and inflammation. Well-known

7 antioxidant and anti-inflammatory mechanisms contribute to the beneficial effects of LSF.

8 Fourier transform infrared microspectroscopy revealed altered composition of macromolecules,

9 including lipids, following OVA-sensitization, which were restored by LSF. RNA sequencing in

10 human peripheral blood mononuclear cells highlighted the anti-inflammatory signature of LSF.

11 Novel findings indicated that LSF reduced the expression and activity of histone deacetylase 8.

12 Further, LSF resulted in histone and $\alpha$-tubulin hyperacetylation in vivo. More generally, this

13 study identified new epigenetic regulatory mechanisms accounting for the protective effects and

14 provide support for the potential clinical utility of LSF in allergic airways disease. 
2 Sulforaphane (SFN) is a dietary isothiocyanate released from the precursor glucoraphanin by the

3 action of plant myrosinase or gut microbiota $\beta$-thioglucosidases ${ }^{1,2,3}$. Myrosinase hydrolyzes the

$4 \quad \beta$-thioglucoside bond from glucosinolate substrates, producing glucose, sulfate, and an unstable

5 aglycone intermediate ${ }^{4,5}$. The aglycone intermediate can be rearranged to form a number of

6 different products including isothiocyanates and nitriles ${ }^{4,5}$. In addition to SFN, the myrosinase-

7 dependent hydrolysis of glucoraphanin yields SFN-nitrile ${ }^{4}$. Sulforaphane is metabolized through

8 the mercapturic acid pathway ${ }^{6}$. The first step involves glutathione conjugation (SFN-GSH)

9 mediated by glutathione S-transferase (GST) enzymes ${ }^{7,8}$. Glutathione conjugates are cleaved

10 further to generate the cysteinylglycine conjugate (SFN-Cys-Gly), cysteine conjugate (SFN-

$11 \mathrm{Cys}$ ), and $N$-acetylcysteine conjugate (SFN-NAC), catalyzed by $\gamma$-glutamyltranspeptidase,

12 cysteinylglycinase, and $N$-acetyltransferase ${ }^{7,8}$.

14 Sulforaphane has been widely investigated in numerous human models of disease including 15 cancer chemoprevention, metabolic disorders, and neurological conditions ${ }^{9,10,11}$. Further, SFN 16 has been studied in numerous preclinical and clinical models of lung damage and airway diseases $1712,13,14,15$. The lack of definitive findings from clinical studies to date, most likely reflects issues 18 with extract preparations and dosage regimes ${ }^{16,17,18}$. A notable exception to this is the beneficial 19 effects of SFN in airborne pollution studies ${ }^{19,20}$. Mercapturic acids are detoxification products

20 formed from GST-catalyzed reactions that are being used in clinical trials as biomarkers of 21 exposure to environmental and industrial chemicals ${ }^{19,20,21}$. Sulforaphane has been shown to 22 enhance the detoxification of toxic and carcinogenic airborne pollutants by increasing the 23 elimination of mercapturic acids ${ }^{19,20}$. 
2 A multitude of mechanisms of action, predominantly involving activation of cellular antioxidant

3 and anti-inflammatory pathways, have been associated with the beneficial effects of SFN in

4 models of disease. It is well-known that SFN activates nuclear factor erythroid 2-related factor 2

5 (Nrf2) and in response to cellular stress, this transcription factor translocates into the nucleus and

6 binds to the antioxidant response element (ARE) ${ }^{18,22}$. The interaction of Nrf2 and coactivator

7 proteins with ARE results in the induction of phase II detoxification and antioxidant enzymes,

8 such as heme oxygenase-1 (HO-1), NAD[P]H:quinone oxidoreductase-1 (NQO1), superoxide

9 dismutase (SOD), catalase (CAT), and glutathione (GSH) ${ }^{22}$. Under basal conditions, Nrf2 is

10 sequestered in the cytosol by Kelch-like ECH-associated protein 1 (Keap1) ${ }^{22}$. Recently it has

11 been shown that LSF causes a reduction of pro-inflammatory cytokine and chemokine

12 production, and alleviates inflammation by targeting antigen-presenting cells ${ }^{23}$.

14 Here, we investigated the effects of administration of L-sulforaphane (LSF) in a murine model of 15 ovalbumin (OVA)-induced chronic allergic airways disease (AAD). This model of chronic AAD 16 recapitulates several features of human asthma including airway inflammation, airway

17 remodeling and airway hyperresponsiveness (AHR) ${ }^{24,25,26}$. Apart from conventional histological 18 and immunohistological analyses we performed genome-wide mRNA-Seq to confirm the anti19 inflammatory signature of LSF in human peripheral blood mononuclear cells (PBMC).

20 Furthermore, we examined epigenetic mechanisms associated with LSF by investigating histone

21 deacetylase enzyme (HDAC) expression and histone acetylation status following LSF

22 administration both in vitro and in the animal model. 


\section{Results}

2 Protective effects of L-sulforaphane in the ovalbumin-induced model of allergic airways

3 disease

4 We used the well-established OVA-induced chronic model of AAD to investigate protection and

5 reversal of pathological features associated with OVA-sensitization by LSF (Figs 1A, 1B and

6 2A). As a positive HDAC inhibitor control, we also explored the protective effects of the FDA

7 approved HDAC inhibitor suberoylanilide hydroxamic acid (SAHA) (Fig 1A) ${ }^{27}$. Using

8 hematoxylin and eosin (H\&E) staining, we observed epithelial thickening, increased goblet cell

9 metaplasia and inflammatory cells peripheral to the bronchial epithelium and basement

10 membrane in sensitized and challenged mice (OVA-vehicle; OVA-VEH) (Fig 1C). The

11 membrane apoptotic marker, Annexin V, was used to assess for apoptosis in the bronchial

12 epithelium (Fig 1D), and morphometric analysis was used to examine epithelial (Fig 1E) and

13 subepithelial thickness (Fig 1F). These findings highlighted the effects of OVA-sensitization

14 and challenge in the OVA-VEH group compared to the saline control mice. LSF attenuated the

15 pathological features associated with OVA-VEH sensitization, whereas SAHA was not effective 16 in this model (Fig 1G).

\section{L-sulforaphane reverses ovalbumin-induced allergic airways disease}

Histological staining was performed to assess for structural changes including epithelial

20 thickness, goblet cell metaplasia and inflammatory infiltrate (Fig 2Bi). Similar to the prevention

21 model we observed goblet cell metaplasia and inflammatory cell infiltrate in the OVA-VEH

22 sensitized and challenged mice compared to saline control mice and mice treated with LSF. An 
1 array of histological stains was performed to assess collagen and reticular fibers in the reversal

2 model (Fig 2B).

4 Masson's trichrome stain was used to highlight the overall change in collagen deposition and 5 changes to epithelial and subepithelial thickness highlighting the beneficial effects of LSF (Fig

6 2Bii). Silver impregnation of collagen and reticular fibers showed weak staining in the saline-

7 and LSF-treated mice in comparison to the OVA-VEH group (Fig 2Biii). Gomori's aldehyde-

8 fuchsin was used to highlight elastic fibers as seen in the fibers surrounding the blood vessels

9 (Fig 2Biv). We observed an increase in elastic fibers surrounding the inner and outer walls of the 10 smooth muscle in the OVA-VEH sensitized mice that was not present in the saline control or

11 mice treated with LSF. Picrosirius red staining was used to show the differential changes in type

12 I and type III collagen deposition to determine the efficacy of LSF treatment in reducing the

13 deposition of type I collagen observed in sensitized airways (Fig 2Bv). Morphometric analysis of

14 Masson's trichrome-stained airways showed LSF significantly attenuated the increases in

15 epithelial thickness (Fig 2C) and subepithelial thickness (Fig 2D). Analysis of sirius red-stained

16 airways showed collagen type III deposition was not affected in the OVA-VEH sensitized mice

17 (Fig 2E). In contrast, collagen type I was significantly increased following OVA-VEH

18 sensitization compared to saline control mice. Treatment of sensitized mice with LSF

19 significantly reduced the augmentation of collagen type I. To assess AHR, mice were challenged

20 with nebulized methacholine (MCh), and the change in resistance from baseline was measured

21 by invasive plethysmography (Fig 2F). MCh-induced AHR was significantly elevated in OVA-

22 VEH mice at doses above $12.5 \mathrm{mg} / \mathrm{ml} \mathrm{MCh}$ compared to saline mice. These effects were 
1 significantly attenuated by LSF mice at doses above $25 \mathrm{mg} / \mathrm{ml} \mathrm{MCh} \mathrm{compared} \mathrm{to} \mathrm{the} \mathrm{OVA-VEH}$

2 sensitized mice.

3

4 Molecular mechanisms associated with protection and reversal of ovalbumin-induced

5 allergic airways disease by L-sulforaphane

6 Immunohistochemical staining of $\alpha$-smooth muscle actin ( $\alpha$-SMA) was used to identify

7 activation of the fibrotic pathway (Fig 3Ai). The results showed that $\alpha$-SMA stained

8 myofibroblasts in the lamina propria was significantly elevated in the OVA-VEH sensitized mice

9 compared to saline control mice. Immunohistochemical images were quantitated to represent

10 myofibroblasts per $100 \mu \mathrm{m}$ of basement membrane length (Fig 3Aii). The increases in the

11 myofibroblasts observed in the OVA-VEH sensitized mice were significantly reduced by

12 treatment with LSF. Downregulation of the expression of Caveolin-1 in OVA-VEH sensitized

13 mice was evident by immunohistochemistry (Fig 3Bi). Analysis of the total area of Caveolin-1

14 expression highlights the significant decrease in OVA-VEH sensitized mice compared to the

15 saline controls and OVA-LSF treated mice (Fig 3Bii). We further assessed airway contraction to

$16 \mathrm{MCh}$ in precision cut lung slices from OVA-challenged mice exposed to LSF ex-vivo.

17 Representative traces measuring the percentage of the airway lumen from the initial area in lung

18 slices from the OVA-VEH mice showed bronchial contraction following perfusion of MCh at a

19 dose of 300nM (Fig 3C). Directly following a five-minute perfusion of MCh to induce airway

20 narrowing, LSF was perfused for the same period. Airway dilation was achieved after

21 approximately one minute of perfusion, in a concentration-dependent manner up to $15 \mu \mathrm{M}$ LSF

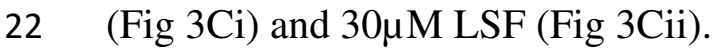


1 Focal plane array-Fourier transform infrared (FPA-FTIR) microspectroscopy was used to

2 investigate changes in spatial distribution of the lipid compositions. As shown in the chemical

3 images (Fig 3Di), lung sections of the OVA-sensitized mouse presented higher intensities of

4 lipids in the bronchial epithelium, compared to those observed for the saline and OVA-LSF

5 mice. The average absorbance and second derivative spectra were calculated from spectra taken

6 from the bronchial epithelium (Figs 3Dii and 3Diii). The derivatization process, specifically

7 second derivatization, has been widely used in spectroscopic analysis because this mathematical

8 approach not only eliminates the baseline effect, but also allows accurate detection and positive

9 identification of band components that are hidden in the presence of broad overlapping spectral

10 features. In this study, absorbance and second derivative spectra were used together for

11 comparing specific lipid bands in the high-wavenumber region. The main spectral features in the

12 high-wavenumber region include the broad $v(\mathrm{O}-\mathrm{H})$ stretching band centred at $\sim 3285 \mathrm{~cm}^{-1}$, and

$13 v\left(\mathrm{CH}_{2} / \mathrm{CH}_{3}\right)$ stretching modes from methylene/methyl groups of lipids, which are presented as

14 triplet bands within 3000-2800 $\mathrm{cm}^{-1}$ spectral range. By a comparison, the $v(\mathrm{O}-\mathrm{H})$ stretching band

15 was shifted from $3290 \mathrm{~cm}^{-1}$ in the saline-treated control mice, to 3292 and $3284 \mathrm{~cm}^{-1}$ in the

16 OVA-VEH and OVA-LSF mice groups respectively, suggesting that OVA treatment caused an

17 alteration of intra- and intermolecular H-bonding in the lung tissue. There was no significant

18 peak shift observed for the $v\left(\mathrm{CH}_{2} / \mathrm{CH}_{3}\right)$ stretching modes. However, the FPA-FTIR images of

19 lipids clearly reveal changes in the intensity of these $v\left(\mathrm{CH}_{2} / \mathrm{CH}_{3}\right)$ absorption peaks, showing a

20 decrease in lipid components in the OVA-LSF mice, and an increase of the lipid components in

21 the OVA-VEH mice when compared with the saline group (Fig 3Di).

22

23 L-sulforaphane modulates key antioxidant defense pathways 
1 To confirm LSF as an inducer of phase II detoxifying enzymes, immunofluorescence was used to

2 detect the presence of Nrf2 in the prevention and reversal models (Fig 4A). Strong staining of

3 Nrf2 was observed in both models in OVA-LSF treated mice. Analysis of the total fluorescence

4 (FL) and foci formation count highlighted significant increases on OVA-LSF treated mice in the

5 prevention (Figs 4Bi and 4Bii) and reversal model (Figs 4Biii and 4Biv). To investigate the

6 localization of Nrf2 foci, line scan analysis of 4',6-diamidino-2-phenylindole (DAPI) and Nrf2

7 staining patterns was performed (Fig 4C). Nrf2 was found in areas of strong DAPI staining

8 associated to heterochromatin in saline and OVA-VEH sensitized mice and elevated staining

9 sometimes outside of heterochromatic regions was seen in OVA-LSF treated mice. The

10 expression of the antioxidant superoxide dismutase 2 (SOD2) was assessed using

11 immunohistochemistry in the reversal model and OVA-LSF reversed OVA-induced reduction in

12 the expression levels (Fig 4D). Expression of heme oxygenase-1 (HO-1) was investigated via

13 immunofluorescence in both models (Fig 4Ei). These findings indicated that LSF treatment of

14 OVA-sensitized mice could reverse the significant elevation seen in OVA-VEH mice in both the 15 prevention (Fig 4Eii) and reversal models (Fig 4Eiii).

17 L-sulforaphane suppresses cytokine and chemokine secretion and downregulates proinflammatory genes

19 Histologically (H\&E)-stained lung sections were analyzed for inflammatory cell infiltrate. OVA-

20 sensitization resulted in a significant increase in inflammation compared to saline control mice.

21 In both models, LSF-treated mice had significantly lower inflammation scores compared with

22 OVA-VEH mice (Fig 5A). 
2 We used immunohistochemistry to determine if LSF would affect the expression of pro-fibrotic

3 genes via the TGF- $\beta /$ smad2 pathway. We showed LSF significantly increased Smad 2 expression 4 compared to saline and OVA-VEH mice (Fig 5B).

5

6 We investigated cytokine and chemokine production from human PBMCs treated with or

7 without LSF $(15 \mu \mathrm{M}, 30 \mu \mathrm{M})$ and $10 \mu \mathrm{M}$ SAHA for 24 hours using a multiplex bead assay (Fig

8 5C). We showed LSF at both concentrations $(15 \mu \mathrm{M}, 30 \mu \mathrm{M})$ significantly attenuated the

9 production of all cytokines and chemokines tested, while SAHA only significantly decreased the 10 secretion of IL-1 $\beta$ (Fig 5C).

12 To investigate whether these findings were reflected in the transcriptome, RNA-sequencing

13 libraries were constructed using total RNA obtained from PBMCs from healthy donors $(\mathrm{n}=4)$

14 following ex vivo treatment with or without $15 \mu \mathrm{M}$ LSF for 24 hours (Fig 5D). Differentially

15 expressed (DE) genes were determined using Deseq2 ( $n=4$, LSF : no LSF) and subjected to gene

16 set enrichment analysis (GSEA) of GO gene sets, which were ranked by effect. Of the top 50

17 ranked gene sets shown (Fig 5Di), only six were upregulated, while 44 sets were downregulated.

18 We additionally filtered differential genes (FDR < 0.05) from the top 100 gene sets for key terms

19 including chemokine, cytokine, immune and inflammation, all of which were predominately

20 downregulated and most notable for $C C L$ and $C X C L$ chemokines (Fig 5Dii). Genes encoding the

21 proteins investigated in Figs 5B and 5C are also highlighted as Selected Genes, which include:

22 TNF (TNF $)$, SMAD2, IL6, IL1B (IL-1 $\beta), C X C L 8(I L-8)$ and CCL4 (MIP-1 $\beta)$. 

hyperacetylation of histone and non-histone proteins

3 To evaluate the epigenetic effects of LSF in airways, immunofluorescence was used to assess the 4 acetylation status of histones (H2B, H3 \& H4) and non-histone protein $\alpha$-tubulin (Figs 6A, B, 5 and C). The findings indicated that LSF administration resulted in hyperacetylated histone H2B

6 in both the reversal and prevention models (Fig 6B) and hyperacetylated H3, H4 histones and $\alpha$ -

7 tubulin in the reversal model (Fig 6C). Furthermore, we explored the expression of HDAC8

8 using immunofluorescence in the prevention model and the results show that HDAC8 expression

9 was significantly elevated in OVA-VEH sensitized mice compared to saline mice and OVA-LSF

10 treated mice (Fig 6D). To further explore the effects of LSF on HDAC8, direct enzymatic

11 inhibition assays indicated LSF selectively inhibited HDAC8 activity $\left(\mathrm{EC}_{50}=92.07 \pm 7.45 \mu \mathrm{M}\right)$

12 (Fig 6E). Immunoblot analysis of class I and class II HDAC enzyme expression in A549 lung

13 cells treated with or without $15 \mu \mathrm{M}$ LSF, further validated that LSF decreased HDAC8

14 expression, and increased acetylation of $\alpha$-tubulin and histone H3 (Fig 6F). The broad spectrum

15 HDAC inhibitor, SAHA $(10 \mu \mathrm{M})$ was used a positive control highlighting the comparatively modest HDAC inhibitory effects of LSF.

We used the molecular docking program, AutoDock Vina, to propose energetically favorable binding sites for LSF on the human class I HDACs: HDAC1, HDAC2, HDAC3 and HDAC8 ${ }^{28}$.

20 In particular, we sought to identify binding modes unique to HDAC8 that may explain its

21 susceptibility to inhibition by LSF compared to the other three members of the family. For

22 docking to human HDAC8, we selected three structures that exemplified three distinct

23 conformations due to the flexible loop B residues S30-K36, adjacent to the catalytic zinc ${ }^{29}$. 
1 These were named 1 pocket (loop B closed, resulting in a single, primary binding pocket, PDB

2 ID: 1T67); 2 pockets (loop B partially opened, with the presence of a secondary pocket adjacent

3 to the primary HDAC pocket, PDB ID: 1T64); and 1 groove (loop B fully opened, with the two

4 pockets merged, PDB ID: 1VKG) ${ }^{29}$. Alignment of these HDAC8 structures is shown in Figs 6Gi

5 and Gii, with loop B highlighted for comparison. For the 1 pocket (loop B closed) structure,

6 docking calculations predicted binding at the primary zinc pocket (Figs 6Giii and Giv) and at a

7 distant site, labelled external-1, both of which also existed in HDAC1-3. A novel site, labeled

8 tertiary, was predicted to bind LSF to HDAC8, which was not predicted for HDAC1-3. This

9 pocket was enclosed by the helix-loop B-helix structural motif spanning residues 21-49, with

10 LSF forming steric contacts with an aromatic cage of residues composed of W137, 141 and 315;

11 Y18 and 20; and a salt-bridge interaction between residue R37 and LSF (Fig 6Gv). Inspection of

12 the docking solutions for predicted LSF binding to other HDAC8 conformations revealed that a

13 number of sub-sites existed in the neighborhood of the novel tertiary site (Fig 6Gvi). Compared

14 to HDAC1-3, the loop B helix region of HDAC8 was shorter by two residues, as shown in the

15 example sequence alignment between HDAC3 and 8 (Fig 6vii). This led to a wider pocket that

16 accommodated binding of LSF to the tertiary site of HDAC8, which was otherwise occluded by

17 the two extra residues as exemplified in HDAC3 (Fig 6Gvii).

18

19 Overall, our findings highlighted the protective effects of LSF in the OVA-induced model of

20 chronic AAD. Mechanisms involving antioxidant and anti-inflammatory pathways account for

21 the beneficial effects of LSF. LSF downregulated the expression and preferentially inhibited

22 HDAC8. Furthermore, hyperacetylation of core histones and $\alpha$-tubulin following administration 
1 in vivo represented key epigenetic mechanisms associated with the effects of LSF in the model of

2 chronic AAD investigated (Fig 7).

3

\section{Discussion}

5 We examined the effects of LSF in the OVA-induced murine model of chronic AAD, and LSF

6 was found to prevent and reverse the pathological features associated with the model (Figs 1 and

7 2). The OVA-induced murine model is well characterized and recapitulates several hallmarks of

8 chronic asthma ${ }^{24,26,30}$. Our findings highlighted that the key features associated with the model,

9 including epithelial thickening, goblet cell metaplasia, apoptosis in the bronchial epithelium, and

10 inflammatory cell infiltration were prominent in OVA-VEH sensitized mice. In the reversal

11 model, LSF treatment significantly reduced the increase in collagen type I deposition and

12 connective tissue fibers that were observed in OVA-VEH sensitized mice (Fig 2). Likewise, LSF

13 significantly reduced the expression of $\alpha$-SMA in myofibroblasts in the lamina propria,

14 attenuated the expression of Caveolin-1 induced by OVA, and significantly increased the

15 expression of phosphorylated Smad2 (Fig 5). In accordance with previous studies, our findings

16 indicated that LSF protected from MCh-induced increases in airway resistance both in vivo (Fig

17 2), and ex vivo (Fig 3) ${ }^{12}$.

19 The antioxidant effects of LSF involving activation of phase II detoxification pathways have

20 been widely investigated ${ }^{12}$. Additionally, the molecular mechanisms that mediate crosstalk

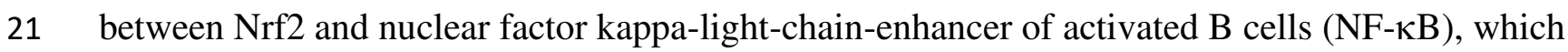

22 regulate oxidative stress and inflammation, have been explored ${ }^{16,17,31}$. Our findings

23 demonstrated that LSF treatment resulted in the modulation of Nrf2 (Fig 4). A significant 
1 increase in the overall expression of Nrf2 and Nrf2 foci was observed in OVA-LSF treated mice

2 and DAPI staining showed that Nrf2 was predominantly localized in heterochromatic regions

3 (Fig 4). This is in line with recent findings, which associated Nrf2 with DNA damage response

4 pathways highlighting the nuclear accumulation of Nrf2 and formation of foci at sites of DNA

5 damage ${ }^{32,33}$.

7 An interesting finding was the significant overexpression of HO-1 in OVA-sensitized mice,

8 which was diminished by administration of LSF in both the prevention and reversal models (Fig

9 4). Expression of HO-1 is significantly upregulated in response to a wide variety of stimuli

10 causing oxidative stress ${ }^{34}$. Investigation of the canonical pathway indicated that the expression

11 of HO-1 was modulated by Nrf2. Our findings indicated that sensitization of mice with OVA

12 resulted in the overexpression of $\mathrm{HO}-1$, which was most likely independent of the Nrf2 pathway.

13 A probable explanation was that OVA-sensitization created oxidative stress releasing stimuli that

14 may have inhibited the transcriptional repressor $\mathrm{BACH} 1$, resulting in increased expression of

15 HO-1 (Fig 4). It is understood that inhibition of BACH1 is sufficient for activation of HO-1

16 irrespective of the status of $\mathrm{Nrf} 2^{34}$. This OVA-sensitization mediated overexpression of HO-1

17 was consistent with previous findings in the context of asthma ${ }^{35}$. Therefore, we can assume that

18 LSF ameliorates oxidative stress stimuli induced by OVA, which cause the overexpression of

19 HO-1. Consistent with activation of the Nrf2 pathway, LSF elevated the expression of the major

20 detoxification enzyme SOD2, which was significantly diminished by OVA-sensitization

21 compared to control mice (Fig 4).

22 
1 Our findings indicated reductions of inflammatory infiltrates in the in vivo model studied (Fig 5).

2 In human PBMC we showed significant reductions in pro-inflammatory cytokines and

3 chemokines following LSF treatment. This included IL-1 $\beta$, IL-6, TNF- $\alpha$, IL-8, IP-10, and MIP-

$4 \quad 1 \beta^{23,36}$. We compared cytokine and chemokine release with the FDA approved pan-HDAC

5 inhibitor SAHA in PBMC cells ${ }^{37}$. Apart from IL-1 $\beta$, there was no significant modulation of

6 other cytokine or chemokine secretion by SAHA at the concentration $(10 \mu \mathrm{M})$ investigated (Fig

7 5). Furthermore, in gene set enrichment analysis of RNA-Seq data in human PBMC, 44 gene sets

8 largely related to the immune response were downregulated and six gene sets were upregulated

9 following treatment with LSF. These findings were consistent in all four samples. Genes

10 encoding for the expression of cytokines and chemokines such as $I L-6, I L-1 A, I L-1 B, E B 13$,

11 CXCL5, and CXCL1, were primarily downregulated, highlighting the anti-inflammatory

12 signature of $\operatorname{LSF}^{23,36,38}$.

14 A particularly interesting observation was the increase in lipid deposition in the bronchial epithelium of OVA-VEH sensitized mice, as indicated by FPA-FTIR chemical images and

16 spectral analysis (Fig 3). Increased wall thickness has been shown to exacerbate airway

17 resistance and more recently, fat-associated airway remodeling has been identified as a potential

18 contributing factor ${ }^{39}, 40$. The relationship between adipose tissue within the airway wall and body

19 mass index (BMI) in non-asthmatics versus asthmatics has been examined ${ }^{39}$. White adipose

20 tissue was present in the outer airway wall in all subject groups and was predominantly found in

21 large to medium sized airways ${ }^{40}$. Our FPA-FTIR data revealed that there was a significant

22 modulation of lipid components following LSF administration with lipid compositions in 
1 airways resembling those of the control group, which was distinct from the OVA-VEH sensitized

2 mice (Fig 3).

4 Epigenetic mechanisms, predominantly chromatin modifications involving the acetylation-

5 deacetylation axis, have been implicated in numerous disease states including asthma and

6 chronic obstructive pulmonary disease ${ }^{41,42,43,44}$. One of the main mechanisms described is the

7 downregulation of the HDAC2 enzyme resulting in aberrant acetylation of the glucocorticoid

8 receptor and resistance to corticosteroid therapy ${ }^{45}$. This may call the potential clinical utility of

9 HDAC inhibitors in AAD into question. However, HDAC inhibitors have well-known

10 pleiotropic properties including modulating inflammatory and antioxidant pathways, and have

11 shown beneficial effects in numerous models of disease $46,47,48,49,50,51$. This includes studies

12 with the prototypical hydroxamic acid Trichostatin A and short-chain fatty acid, valproic acid, in

13 models of AAD 52,53,54,55. Here, unlike Trichostatin A, we show that the hydroxamic acid

14 HDAC inhibitor SAHA was not effective in modulating airway responses in our model, further

15 highlighting the complexities associated with the use of pleiotropic pan-HDAC inhibitors in 16 airways disease (Fig 1).

17

18 Our findings indicated that LSF modulated the acetylation of histones $2 \mathrm{~B}$ and $\mathrm{H} 3$, and $\alpha$-tubulin 19 in the mouse model studied (Fig 6). This, in accordance with previous work, has indicated that 20 SFN possesses HDAC inhibitor activity in vitro and in in vivo ${ }^{56,57,58}$. Using in vitro enzymatic 21 assays and in A549 human alveolar epithelial cells, we show that LSF preferentially inhibited the 22 HDAC8 enzyme with very modest activity in comparison to SAHA. As described, steric 
1 hindrance may preclude relevant binding of LSF to other class I HDAC enzymes (Fig 6). Indeed,

2 we performed independent experiments using cell-free assays indicating that LSF did not inhibit

3 any of the other metal-dependent HDAC enzymes at biologically relevant concentrations (mM

4 range for HDAC3, HDAC6, and HDAC7 and undetectable for others). This is an interesting

5 observation as it has been previously shown that a potent specific HDAC8 inhibitor (PCI-34501),

6 attenuated airway responses in OVA-sensitized mice ${ }^{59}$.

8 A limitation of our study is that the potential effects of key SFN metabolites were not explicitly

9 investigated. We have recently shown that although not as potent as LSF, metabolites such as

10 SFN-Cys and SFN-NAC, have important roles in anti-inflammatory pathways ${ }^{23}$. Here, we show

11 acetylation of $\alpha$-tubulin following in vivo administration of LSF (Fig 6). Given that acetylation

12 of $\alpha$-tubulin is regulated by inhibition of HDAC6, and our cell-free assays highlight that LSF

13 preferentially inhibits HDAC8, it presumed that an LSF metabolite mediates this effect ${ }^{60}$.

14 According to previous observations, the metabolite that most likely possesses HDAC inhibition

15 activity is SFN-Cys ${ }^{61,62,63}$. Therefore, it is worthy to systematically investigate the biological

16 effects and bioactivity of SFN metabolites. However, these considerations do not detract from

17 our findings which demonstrate accumulation of hyperacetylated histones and $\alpha$-tubulin

18 following administration of LSF in vivo (Fig 6).

20 Overall, our findings confirmed the efficacy of LSF in attenuating pathologies associated with

21 AAD, involving activation of antioxidant and anti-inflammatory pathways (Fig 7). Preferential

22 inhibition of HDAC8 by LSF and accumulation of acetylated core histones and $\alpha$-tubulin in vivo 
1 following LSF administration represented a new epigenetic regulatory mechanism. Sulforaphane

2 and high glucoraphanin extracts have had mixed results in human trials and currently, there are

3 no therapeutic uses of SFN or extracts in the clinic. However, our findings along with

4 accumulated evidence, highlight the clinical potential of SFN as either a prophylactic or a

5 therapeutic in the context of AAD. A pharmaceutical grade stable form of LSF or optimized

6 glucoraphanin-rich extracts may enable clinical translation.

8 Materials and Methods

9 Chemicals. In animal studies, LSF (Santa Cruz Biotechnology, Dallas, Texas, USA) was used at 10 a dose of $5 \mathrm{mg} / \mathrm{kg}$ dissolved in $1 \%$ (v/v) dimethyl sulfoxide (DMSO, Sigma-Aldrich, St. Louis, 11 MO, USA) in 0.9\% (v/v) normal saline solution (Baxter Health Care, NSW, Australia) and

12 SAHA (Sigma) was used at $100 \mathrm{mg} / \mathrm{kg}$ in $1 \%(\mathrm{v} / \mathrm{v})$ DMSO in $0.9 \%(\mathrm{v} / \mathrm{v})$ saline. The selected 13 doses are known to be biologically active and have been used in experimental murine models of 14 disease without significant toxicity ${ }^{64,65}$. Chemical structures of LSF and SAHA are shown in 15 Fig 1A. For in vitro studies, LSF, SAHA, and MCh were all purchased from Sigma. LSF, and 16 SAHA were dissolved in $100 \%$ (v/v) DMSO and stored as stock solutions of $20 \mathrm{mM}\left(-80^{\circ} \mathrm{C}\right)$, $1710 \mathrm{mM}\left(-20^{\circ} \mathrm{C}\right)$ and $1 \mathrm{M}\left(-80^{\circ} \mathrm{C}\right)$, respectively.

19 Animals. Six-week-old female BALB/c mice obtained from Walter and Eliza Hall Institute 20 Bioservices (prevention model; Parkville, VIC, Australia) and Monash Animal Services (reversal 21 model; Clayton, VIC, Australia). Mice were housed under specific pathogen-free conditions, 22 maintained at $21^{\circ} \mathrm{C}, 12$-hour light/dark cycle, fed standard laboratory chow (Barastoc Stockfeeds, 
1 Pakenham, VIC, Australia), and water ad libitum. The BALB/c mice strain was chosen for their

2 strong Th2 responses in OVA-induced AAD models ${ }^{66}$. Due to their increased sensitivity to

3 OVA and AAD models, female mice were chosen for this study ${ }^{66,67}$. The prevention model

4 experimental protocol was approved by the Murdoch Children's Research Institute Animal

5 Ethics Committee (approval no. A597). The reversal model experimental protocol was approved

6 by the Monash University Animal Ethics Committee (MARP/2012/085). All mice were provided

7 an acclimatization period of 4-5 days before any experimentation. All experimental procedures

8 followed the Australian guidelines for the care and use of laboratory animals for scientific

9 purposes.

11 Mouse model of chronic allergic airways disease. To assess the effects of LSF in chronic AAD

12 two models were used to determine protective effects and ability to reverse AAD in an

13 established model of OVA-induced chronic AAD. This model was chosen for its pathological

14 similarities to human asthma, including increased allergic responses indicated by increased

15 immunoglobulin E against OVA (OVA-specific IgE), AHR and remodeling changes such as

16 epithelial remodeling, goblet cell metaplasia, and subepithelial collagen deposition (fibrosis).

17 Treatment groups were divided into the prevention and reversal models according to the

18 timelines shown in Figs 1B and 2A. Detailed protocols of the prevention model ${ }^{52}$ and reversal

19 model ${ }^{68}$ have been described previously.

20

21 Prevention model. Briefly, mice were sensitized with two intraperitoneal (i.p) injections of $10 \mu \mathrm{g}$

22 of grade V chicken egg OVA (Sigma) and 1mg of aluminum potassium sulphate adjuvant (alum)

23 (Sigma) in $0.5 \mathrm{~mL}$ of $0.9 \%(\mathrm{v} / \mathrm{v})$ saline, which was subsequently administered via i.p on days 0 
1 and 14. Mice were then challenged by whole body inhalation exposure (nebulization) to

2 aerosolized $2.5 \%(\mathrm{w} / \mathrm{v})$ OVA in saline for 30 minutes, three days per week for six weeks using

3 an ultrasonic nebulizer (NE-U07, Omron Corporation, Tokyo, Japan), between days 21 and 63 to

4 establish AAD. Following exposure to nebulized OVA, mice were treated with 5mg/kg LSF

5 (OVA-LSF) or 100mg/kg SAHA (OVA-SAHA, n=6) or vehicle control (OVA-VEH, n=15) by

6 i.p injection, three days per week for six weeks, which represent doses known to be effective and

7 non-toxic ${ }^{30,44}$. A fourth group of mice were sensitized with $1 \mathrm{mg}$ alum in $0.5 \mathrm{ml}$ saline on days 0

8 and 14, and were challenged with saline aerosols three days per week for six weeks $(\mathrm{n}=15)$. Mice

9 were euthanized with an i.p injection of ketamine and xylazine $(200 \mu \mathrm{g} / \mathrm{g}: 10 \mu \mathrm{g} / \mathrm{g}) 4$ days after

10 the last nebulization, on day 67.

11

12 Reversal model. Saline control mice $(n=6)$ and vehicle control mice (OVA-VEH, $n=6)$ were

13 sensitized and nebulized to saline or OVA as described in the prevention model. Mice were

14 treated with saline or $5 \mathrm{mg} / \mathrm{kg}$ LSF (OVA-LSF, $\mathrm{n}=8$ ) by i.p injection 24 hours after the last OVA

15 nebulization five times every 72 hours between days 64 and 76. Mice were euthanized by i.p

16 injection of ketamine and xylazine $(200 \mu \mathrm{g} / \mathrm{g}: 10 \mu \mathrm{g} / \mathrm{g}) 48$ hours after the last treatment on day

1778.

18

19 Measurement of airway reactivity in vivo. AHR was assessed by plethysmography, 14 hours

20 after the final treatment with LSF or vehicle using a mouse invasive plethysmograph (Buxco

21 Electronics, Troy, NY). Following anaesthetization using an i.p injection of ketamine and

22 xylazine $(200 \mu \mathrm{g} / \mathrm{g}: 10 \mu \mathrm{g} / \mathrm{g})$, mice were tracheostomized and cannulated via the jugular vein.

23 Mice were ventilated with a small animal respirator (Harvard Apparatus, Holliston, MA) 
1 delivering $0.01 \mathrm{ml} / \mathrm{g}$ bodyweight at a rate of 120 strokes $/ \mathrm{min}$. A baseline airway resistance was

2 recorded (Biosystem XA; Buxco Electronics), then every three minutes after increasing

3 intravenous doses of $\mathrm{MCh}(3.1,6.3,12.5,25,50$ and $100 \mathrm{mg} / \mathrm{ml})$ in phosphate buffered saline.

4 The change in airway resistance from baseline level per dose was recorded in Finepoint (Buxco

5 Electronics).

6

7 Measurement of airway reactivity ex vivo. Preparation of precision cut lung slices and

8 measurement of responses to MCh were performed in OVA-VEH sensitized mice as previously

9 described ${ }^{69,70}$. Briefly, lungs were inflated with warm $2 \%(w / v)$ ultra-pure low melting point

10 agarose (Invitrogen, Carlsbad, CL, USA) in Hanks' balanced salt solution supplemented with 40

11 mM HEPES (HBSS, Sigma), via a tracheal cannula. After solidification of the agarose at $4^{\circ} \mathrm{C}(20$

12 mins), a single lobe was mounted in cold HBSS to a vibratome (VT 1000S, Leica Microsystems,

13 Wetzler, Germany) in preparation for serial sectioning $(150 \mu \mathrm{m})$. Measurements of airway

14 responses to MCh was performed using a gravity-fed system where lung slices were perfused at

15 a constant rate (5 mins) for each condition; $300 \mathrm{nM} \mathrm{MCh}$, increasing doses of $\operatorname{LSF}(3,10,15$ and

$1630 \mu \mathrm{M})$ and HBSS in resting periods.

17

18 Lung histopathology. Lung tissues were dissected, and right lung lobes were fixed with $10 \%$

19 (v/v) neutral buffered formalin (Sigma) for 24 hours, routinely processed and embedded in

20 paraffin. Lung sections $(3 \mu \mathrm{m})$ were assessed by standard histological staining procedures;

21 hematoxylin and eosin (H\&E) for inflammatory cell infiltration, Masson's trichrome for

22 epithelial and subepithelial collagen deposition, silver impregnation for detection of reticular and 
1 collagen fibers, Gomori's aldehyde-fuchsin for elastic fibers and sirius red staining for

2 differentiation of collagen type I and type III.

4 Airway tissue inflammation cell score. Airway inflammatory cell infiltration was determined

5 from $\mathrm{H} \& \mathrm{E}$ stained sections. A minimum of ten airways per mouse were captured using an

6 Olympus FSX100 microscope mounted with a digital camera (Olympus, Tokyo, Japan). The

7 degree of airway inflammatory cell infiltration around the bronchi was scored by two

8 independent blinded investigators using the following score scale: $0=$ no inflammatory cells; $1=$

9 1-3 layers of inflammatory cells; $2=4-6$ layers; $3=7-10$ layers and congestion; or $4=>10$

10 layers of inflammatory cells and severe congestion. For each mouse, a minimum of ten airways

11 were observed and the average scores were taken.

13 Morphometric analysis of structural changes. Epithelial thickness and subepithelial collagen

14 thickness were assessed from Masson's trichrome stained sections. A minimum of ten airways 15 per mouse were captured using an Olympus FSX100 microscope mounted with a digital camera

16 (Olympus). Bronchi measuring between 150-350 $\mu$ m luminal diameter were analyzed using

17 Image Pro-Plus software (v6.0, Media Cybernetics, MD, USA) after calibration with a reference micrometer slide. The thickness of the bronchial epithelial layer was measured by tracing around

19 the basement membrane and the luminal surface of epithelial, and calculating the mean distance

20 between the two. Subepithelial collagen thickness was also measured by tracing around the outer

21 extent of the total collagen layer in the submucosal region, around the basement membrane, and

22 the mean distance between these lines calculated. Differentiation of collagen type I and type III

23 was assessed from sirius red F3B (Sigma) stained slides. Sirius red staining was captured under 
1 polarized light using an Olympus BX61 microscope (Olympus) automated with a FVII digital

2 camera to differentiate between the types of collagen. Type I (red and yellow under polarized

3 light) and type III (green under polarized light) collagen was quantified using ImageJ analysis

4 software (FIJI v1.48a, NIH, Bethesda, MD).

5

6 Immunohistochemistry. Formalin-fixed paraffin embedded sections were used to evaluate the

7 protein expression of $\alpha$-smooth muscle actin ( $\alpha$-SMA) to identify airway smooth muscle,

8 Caveolin-1, superoxide dismutase 2 (SOD2) and phosphorylated Smad2. Primary antibodies:

9 mouse monoclonal anti- $\alpha$ SMA (1:150, Dako, Glostrup, Denmark); rabbit polyclonal anti-

10 Caveolin-1 (1:1000, Santa Cruz Biotechnology); rabbit monoclonal (EPR2560Y) anti-SOD2

11 (1:250, Abcam, Cambridge, UK) and rabbit polyclonal anti-phospho-Smad2 (ser465/467)

12 (1:500, Cell Signaling Technology, Danvers, MA, USA) were bound, biotinylated and detected

13 using streptavidin horseradish peroxidase (Dako). The chromogen 3,3-diaminobenzidine (DAB,

14 Thermo Fisher Scientific, Waltham, MA, USA) was used and sections were counterstained with

15 hematoxylin (Sigma). Images were acquired using the Olympus FSX100 microscope (Olympus),

16 a minimum of five airways were collected per mouse. $\alpha$-SMA was quantified by counting the

17 number of myofibroblasts surrounding the airways per $100 \mu \mathrm{m}$ of basement membrane measured

18 using Image Pro Plus Software (Media Cybernetics). All other antibodies were assessed by

19 measuring the mean area $\left(\mu \mathrm{m}^{2}\right)$ of the protein expressed by detecting DAB staining using Image

$20 \mathrm{~J}$ software (FIJI v1.48a, NIH, Bethesda, MD) incorporating the color deconvolution plugin,

21 which isolates the brown colour channel from the brightfield image. 
1 Immunofluorescence. Formalin-fixed paraffin embedded sections were used to evaluate the

2 protein expression of: membrane apoptotic marker Annexin V; nuclear factor erythroid 2-related

3 factor 2 (Nrf2), which regulates antioxidant proteins like heme oxygenase-1 (HO-1); the

4 acetylated state of histones H2B (H2Bac), H3 (H3ac), H4 (H4ac) and non-histone protein $\alpha$ -

5 tubulin and histone deacetylase 8 (HDAC8) as previously described ${ }^{52}$. Briefly, after

6 deparaffinization and antigen retrieval (Dako), slides were blocked using Superblock (Thermo

7 Fisher Scientific) and exposed to primary rabbit monoclonal antibodies: anti-Annexin V (1:500,

8 Abcam), anti-Nrf2 (1:250, Abcam), anti-HO-1 (1:500, Abcam), anti-histone H2B acetyl (K15)

9 (1:500, Abcam), anti-histone H3 acetyl (K9) (1:500, Abcam), rabbit polyclonal anti-histone H4

10 acetyl (1:500, Millipore) and mouse monoclonal antibodies, anti- $\alpha$-tubulin (1:500, Sigma) and

11 anti-HDAC8 (1:250, Sigma) all diluted in 1\% (v/v) bovine serum albumin (BSA, Sigma).

12 Primary antibodies were conjugated with secondary antibodies, goat anti-mouse Alexa 488

13 (Molecular Probes Invitrogen) and goat anti-rabbit 546 (Molecular Probes) diluted in 1\% (v/v)

14 BSA (1:500) and mounted in Prolong Gold Antifade with DAPI (Invitrogen). Slides were

15 incubated overnight at $4^{\circ} \mathrm{C}$ before imaging. Annexin $\mathrm{V}$ images were acquired using an Olympus

16 BX61 motorized upright fluorescence microscope automated with FVII Camera (Olympus) using

17 a 10x/0.3 and 20x/0.5 U PLAN FL objectives and fluorescence filter cubes used included: DAPI

18 ( $\left.\lambda_{\text {ex }}: 350 / 50, \lambda_{\text {em }}: 460 / 50 \mathrm{~nm}\right)$, FITC ( $\left.\lambda_{\text {ex }}: 470 / 40, \lambda_{\text {em }}: 525 / 50 \mathrm{~nm}\right)$ and TRITC $\left(\lambda_{\text {ex }}: 545 / 30\right.$,

$\left.19 \lambda_{\text {em }}: 620 / 60 \mathrm{~nm}\right)$. All other antibodies were acquired using the Nikon A1R-si resonant scanning

20 confocal system with upright Nikon D-Eclipse microscope fitted with an automated digital

21 camera (Nikon Ti, Tokyo, Japan) in association with Nikon NIS-Elements AR 3.2 64-bit

22 software (NIS Elements Advanced Research, Tokyo, Japan). Samples were captured using a

$2360 x / 1.0 W$ DIC N2 $\infty / 0$ oil 2.8 Nikon Japan NIR Apo oil immersion objective (NA=1.4). Images 
were acquired with sequential excitation: $405 \mathrm{~nm}\left(\lambda_{\mathrm{em}}: 450 / 50\right), 488 \mathrm{~nm}\left(\lambda_{\mathrm{em}}: 490 / 525\right)$ and 568 $\mathrm{nm}\left(\lambda_{\mathrm{em}}: 562 / 95\right)$ laser lines and collected in 512 x 512 pixel format (mono 16-bit) with a $0.5 \mu \mathrm{m}$ step size and $2.2 \mathrm{pixel} / \mathrm{dwell}$. A minimum of five airways per mouse were acquired, and images were processed and analyzed for mean total fluorescence (FL) intensity using ImageJ (FIJI). Nrf2 foci and line scan analysis was assessed using Image J processing tools (FIJI).

\section{Focal plane array-Fourier transform infrared (FPA-FTIR) microspectroscopy. Lung} samples were sectioned $(4 \mu \mathrm{m})$ using a Leica RM 2135 microtome (Leica Biosystems) onto calcium fluoride $\left(\mathrm{CaF}_{2}\right)$ windows (Crystran, Dorset, UK). The sections were subsequently deparaffinized by two consecutive 5 min washes with xylene (Sigma-Aldrich) and stored in desiccator prior to FPA-FTIR microspectroscopy. The acquisition of FPA-FTIR chemical images was performed using an offline FPA-FTIR microspectroscopic system (Bruker Optik GmbH, Ettlingen, Germany) at the Australian Synchrotron Infrared Microspectroscopy (IRM) beamline (Clayton, VIC, Australia) as described previously ${ }^{30}$. Briefly, the FPA-FTIR images were acquired in transmission mode using a Bruker Hyperion 3000 FTIR microscope, with a liquid nitrogen cooled 64 x 64 element FPA detector and a matching 15x objective and condenser (NA $=0.40)$, coupled to a Bruker Vertex 70 FTIR spectrometer with an internal thermal $\left(\mathrm{Globar}^{\mathrm{TM}}\right)$ IR source. Each FPA-FTIR image (4 x 4 grid) was acquired within the 4,000-800 $\mathrm{cm}^{-1}$ spectral region with a sampling area of $180 \times 180 \mu \mathrm{m}^{2}$. The spectral images were collected with $8 \mathrm{~cm}^{-1}$ resolution, 64 co-added scans, Blackman-Harris 3-term apodization, Power-Spectrum phase correction, and a zero-filling factor of two, which were set as default acquisition parameters using the OPUS 7.2 imaging software suite (Bruker). Background spectra were measured using the same acquisition parameters, by focusing on a clean surface area of the $\mathrm{CaF}_{2}$ window without 
any tissue. FPA-FTIR images were pre-processed for an atmospheric compensation, baseline correction (concave rubberband algorithm) and vector normalization. The spatial distribution of lipids were generated based on integrated areas under the lipid region $\left(3,000-2,800 \mathrm{~cm}^{-1}\right)$. Spectra localized to the bronchial epithelium (104 spectra for saline, 156 spectra for OVA-VEH and 114 spectra for OVA-LSF airways) were extracted from the FPA-FTIR images. The extracted spectra were then averaged and converted into its corresponding second derivative using a 25 smoothing point Savitzky-Golay algorithm in OPUS 7.2 software (Bruker).

HDAC8 activity profile. To determine the in vitro HDAC inhibitor capacity of LSF against the metal-dependent HDAC1-11 enzymes, direct fluorescence based enzymatic inhibition assays were performed by Reaction Biology Corp. (Malvern, PA, USA) using the fluorogenic peptides from p53 residues 379-382 (RHKAcKAc), according to the company's standard operating procedures. Two independent experiments were performed and the $\mathrm{EC}_{50}$ values were determined from ten concentrations $(0.01-100 \mu \mathrm{M})$ assayed in three-fold serial dilution. SAHA was used as a reference compound. $\mathrm{EC}_{50}$ values were calculated by fitting the data to the four-parameter logistic equation using GraphPad Prism, v7.01 (GraphPad, San Diego, CA, USA).

Cell culture for in vitro studies. Human peripheral blood mononuclear cells (PBMC) were fractionated using Ficoll Paque (GE Healthcare, Wauwatosa, Wisconsin, USA) from blood samples (healthy participants; $n=4$ ) obtained from the Australian Red Cross Blood Bank (Melbourne, VIC, Australia) under ethics project (\#304/12) approved by the Alfred Hospital Ethics Committee (Alfred Health, Melbourne, VIC, Australia). Cells were harvested fresh on the 
day of experiments and maintained in complete-Roswell Park Memorial Institute (RPMI) 1640 medium supplemented with 20 mmol/L HEPES (pH 7.4; GIBCO-Invitrogen, Carlsbad, CA, USA), $10 \%$ (v/v) fetal bovine serum (FBS, In vitro Technologies, Noble Park, VIC, Australia), 2 $\mathrm{mmol} / \mathrm{L} \mathrm{L}$-glutamine (GIBCO-Invitrogen), and 1\% (v/v) penicillin/streptomycin (GIBCOInvitrogen) at $37^{\circ} \mathrm{C}, 5 \%(\mathrm{v} / \mathrm{v}) \mathrm{CO}_{2}$. Human epithelial lung A549 cells, purchased from the American Type Culture Collection (ATCC, Manassas, VA, USA), were grown as monolayers in Dulbecco's Modified Eagle Medium (DMEM) (Life Technologies, Carlsbad, USA) supplemented with $10 \%(\mathrm{v} / \mathrm{v})$ FBS and $1 \%(\mathrm{v} / \mathrm{v})$ penicillin/streptomycin and maintained in the exponential growth phase under sterile conditions, $37^{\circ} \mathrm{C}, 5 \%$ (v/v) $\mathrm{CO}_{2}$.

Chemokine and cytokine multiplex bead assay. Chemokines and cytokine production from mononuclear cells was measured by multiplex array. PBMCs were incubated with LSF $(15 \mu \mathrm{M}$ and $30 \mu \mathrm{M})$, and SAHA $(10 \mu \mathrm{M})$ or left untreated for 24 hours at $37^{\circ} \mathrm{C}, 5 \%(\mathrm{v} / \mathrm{v}) \mathrm{CO}_{2}$. Supernatants were harvested following centrifugation $(800 \mathrm{~g} ; 10 \mathrm{mins})$ and measured for the presence of IL-1 $\beta$, IL-6, IL-8, IP-10, MIP- $1 \beta$ and TNF- $\alpha$ using the multiplex bead assay (BioRad Laboratories Inc, Hercules, CA, USA) according to the manufacturers' procedures. All supernatants were assayed undiluted, in triplicate. Briefly, a standard curve was prepared using four-fold serial dilutions of the cytokine standards provided using RPMI-FCS. Assay controls were prepared by adding known concentrations of the cytokine standard using a 1:2 and 1:4 dilution in RPMI-FCS. Culture medium was used as a negative control. Anti-cytokine beads and matched anti-cytokine biotinylated reporters were measured on a Luminex 200 Bio-analyzer and data was analyzed in LuminexIS v2.3 software (Luminex Corporation, Austin, TX, USA), and concentrations reported in $\mathrm{pg} / \mathrm{ml}$. 
2 RNA-Seq. PBMCs were cultured with or without $15 \mu \mathrm{M} \mathrm{LSF}$ for 24 hours prior to RNA

3 extraction, which comprised of TRIzol (Invitrogen) cell lysis followed by RNeasy (Qiagen,

4 Hilden, Germany) column purification with on-column DNase treatment. Equal amounts of

5 purified RNA (5mg per sample) were used in the construction of sequencing libraries (RNA-

6 sequencing library preparation kit, Illumina, San Diego, CA, USA). Sequencing of RNA-Seq

7 libraries was performed on a Genome Analyzer IIx with a 36-cycle (v4) sequencing kit

8 (Illumina). Sequence reads were extracted using Pipeline (v1.6) software (Illumina) before

9 alignment (GRCh38 cDNA reference, release-101, Ensembl) and quantification of transcript

10 counts using Kallisto quant (v0.45.0) ${ }^{71}$. Transcript level counts were aggregated to genes, before

11 sample normalization and differential expression analysis with Deseq2 (v1.28.1 ${ }^{72}$. Differential

12 genes were ranked by significance and fold change as described previously ${ }^{51}$, prior to gene set

13 enrichment analysis (GSEA) of gene ontology (GO) gene sets (v7.2) ${ }^{73}$, using 'mitch' (v1.0.8) ${ }^{74}$

14 with mitch settings: priority='effect'. Heat maps and differential expression plots for RNA data 15 were generated using ggplot2 (v3.3.2) ${ }^{75}$.

17 Western blotting. To determine the effects of LSF on the expression of class I and II HDAC 18 enzymes, protein was assessed in A549 cells using immunoblotting techniques as previously 19 described ${ }^{76}$. Briefly, A549 cells were treated with $15 \mu \mathrm{M}$ LSF and 10 $\mu \mathrm{M}$ SAHA for 24 hours

20 prior to cell lysis using mammalian protein extraction reagent (MPER, Thermo Fisher

21 Scientific). Protein was measured using the Bradford assay, fractionated using 4-12\% Bis-Tris

22 SDS-PAGE (Invitrogen) and transferred to nitrocellulose membranes. The membranes were

23 incubated overnight with primary antibodies: polyclonal rabbit anti-HDAC1, HDAC2, HDAC3, 
HDAC4, HDAC6, HDAC8, HDAC9 AND HDAC10 $(2 \mu \mathrm{g} / \mathrm{ml}$; BioVision Inc. Milpitas, CA, USA), rabbit monoclonal anti-histone H3 acetyl (K9) (1 $\mu \mathrm{g} / \mathrm{ml}$, Abcam), mouse monoclonal anti$\alpha$-tubulin (1 $\mu \mathrm{g} / \mathrm{ml}$, Sigma) and mouse monoclonal anti-GAPDH (1 $\mu \mathrm{g} / \mathrm{ml}$, Sigma). The membranes were incubated with horseradish peroxidase-conjugated goat anti-mouse or donkey anti-rabbit secondary antibodies (1:10000, Dako) followed by enhanced chemiluminescence 6 staining (Sigma).

8 Molecular modeling. AutoDock Vina ${ }^{28}$ was used to propose energetically favorable binding 9 sites for LSF on the human class I HDAC enzymes. For docking to human HDAC8, three structures from the RCSB Protein Data Bank (PDB) exemplifying three distinct conformations of HDAC8 co-crystallized with different inhibitors (PDB ID: 1T67, 1T64, 1VKG) were used ${ }^{29}$.

12 Docking to HDAC2 and HDAC3 were performed on PDB ID: 3MAX and 4A69, respectively ${ }^{77}$,

$13{ }^{78}$. For each HDAC structure, the first protein chain (chain A) was isolated, water molecules and 14 other ligands were removed, and the zinc atom was retained for subsequent docking calculations. 15 All HDAC structures were energy-minimized using GROMACS (v4.5.5) ${ }^{79}$ with the GROMOS 16 forcefield and 53A6 parameter set. Each HDAC structure was energy minimized using the

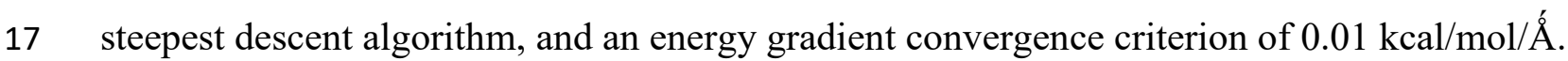

18 The LSF structure was created using ChemDraw (Perkin Elmer, Waltham, MA, USA) and saved 19 in PDB format. Docking calculations were performed using Autodock Vina. All HDAC and LSF 20 PDB files were processed using PyRx ${ }^{80}$ to create their corresponding PDBQT files. All five 21 rotatable torsions for LSF were activated, while HDAC structures were assumed to be rigid. 22 PyRx was used to define the ligand search grid, identical for all HDACs modeled, with 23 dimensions x-size: 77, y-size: 65, and z-size: 60, encompassing the entire protein surface for an 
1 exhaustive search of potential binding sites. The searches were conducted with an exhaustiveness

2 of 2000. The top 10 predicted LSF binding poses for each HDAC model were visualized and

3 analyzed using Visual Molecular Dynamics (v1.9.2) (VMD, Beckman Institute, Urbana, IL,

4 USA).

5

6 Statistics. All data is expressed as mean \pm SEM, where each $n$ represents a single mouse ( $>$ five

7 airways per mouse unless otherwise specified) or a single blood donor $(n=4)$. Groups were

8 compared using a one-way analysis of variance (ANOVA) with a 95\% confidence interval and

9 Tukey post-hoc test where appropriate using GraphPad Prism, v7.01 (GraphPad).

10

11

12

13

14

15

16

17

18

19

20 


\section{References}

1. Zhang Y, Talalay P, Cho CG, Posner GH. A major inducer of anticarcinogenic protective enzymes from broccoli: isolation and elucidation of structure. Proceedings of the National Academy of Sciences of the United States of America 89, 2399-2403 (1992).

2. Prochaska HJ, Santamaria AB, Talalay P. Rapid detection of inducers of enzymes that protect against carcinogens. Proceedings of the National Academy of Sciences of the United States of America 89, 2394-2398 (1992).

3. Fahey JW, Holtzclaw WD, Wehage SL, Wade KL, Stephenson KK, Talalay P. Sulforaphane bioavailability from glucoraphanin-rich broccoli: control by active endogenous myrosinase. PloS one 10, e0140963-e 0140963 (2015).

4. Angelino D, et al. Myrosinase-dependent and-independent formation and control of isothiocyanate products of glucosinolate hydrolysis. Front Plant Sci 6, (2015).

5. Matusheski NV, Jeffery EH. Comparison of the bioactivity of two glucoraphanin hydrolysis products found in broccoli, sulforaphane and sulforaphane nitrile. Journal of Agricultural and Food Chemistry 49, 5743-5749 (2001).

6. Tortorella SM, Royce SG, Licciardi PV, Karagiannis TC. Dietary sulforaphane in cancer chemoprevention: the role of epigenetic regulation and HDAC inhibition. Antioxidants \& Redox Signaling 22, 1382-1424 (2015).

7. Vanduchova A, Anzenbacher P, Anzenbacherova E. Isothiocyanate from broccoli, sulforaphane, and its properties. Journal of Medicinal Food 22, 121-126 (2018).

8. Amjad AI, Parikh RA, Appleman LJ, Hahm E-R, Singh K, Singh SV. Broccoli-derived sulforaphane and chemoprevention of prostate cancer: from bench to bedside. Current Pharmacology Reports 1, 382-390 (2015).

9. Zimmerman AW, et al. Randomized controlled trial of sulforaphane and metabolite discovery in children with Autism Spectrum Disorder. Molecular Autism 12, 38 (2021).

10. Sun Y, et al. Protective effects of sulforaphane on type 2 diabetes-induced cardiomyopathy via AMPK-mediated activation of lipid metabolic pathways and NRF2 function. Metabolism Clinical and Experimental 102, (2020).

11. Singh K, et al. Sulforaphane treatment of autism spectrum disorder (ASD). Proc Natl Acad Sci U $S$ A 111, 15550-15555 (2014). 
1 12. Brown RH, Reynolds C, Brooker A, Talalay P, Fahey JW. Sulforaphane improves the bronchoprotective response in asthmatics through Nrf2-mediated gene pathways. Respiratory Research 16, 106 (2015).

13. Jiao Z, Chang J, Li J, Nie D, Cui H, Guo D. Sulforaphane increases Nrf2 expression and protects alveolar epithelial cells against injury caused by cigarette smoke extract. Molecular Medicine Reports 16, 1241-1247 (2017).

14. Cho H-Y, et al. Sulforaphane enriched transcriptome of lung mitochondrial energy metabolism and provided pulmonary injury protection via Nrf2 in mice. Toxicology and Applied Pharmacology 364, 29-44 (2019).

15. Sudini K, et al. A randomized controlled trial of the effect of broccoli sprouts on antioxidant gene expression and airway inflammation in asthmatics. The Journal of Allergy and Clinical Immunology In Practice 4, 932-940 (2016).

16. Mazarakis N, Snibson K, Licciardi PV, Karagiannis TC. The potential use of 1-sulforaphane for the treatment of chronic inflammatory diseases: A review of the clinical evidence. Clinical Nutrition 39, 664-675 (2020).

17. Houghton CA. Sulforaphane: its "coming of age" as a clinically relevant nutraceutical in the prevention and treatment of chronic disease. Oxidative Medicine and Cellular Longevity 2019, 2716870-2716870 (2019).

18. Fahey JW, Kensler TW. The challenges of designing and implementing clinical trials with broccoli sprouts... and turning evidence into public health action. Front Nutr 8, (2021).

19. Kensler TW, et al. Modulation of the metabolism of airborne pollutants by glucoraphanin-rich and sulforaphane-rich broccoli sprout beverages in Qidong, China. Carcinogenesis 33, 101-107 (2012).

20. Egner PA, et al. Rapid and sustainable detoxication of airborne pollutants by broccoli sprout beverage: results of a randomized clinical trial in China. Cancer prevention research (Philadelphia, Pa) 7, 813-823 (2014).

21. M. De Rooij Jan N. M. Commandeur Nico P. E. Vermeulen BEN. Mercapturic acids as biomarkers of exposure to electrophilic chemicals:applications to environmental and industrial chemicals. Biomarkers 3, 239-303 (1998).

22. Keum Y-S. Regulation of Nrf2-mediated phase II detoxification and anti-oxidant genes. Biomolecules \& Therapeutics 20, 144-151 (2012).

23. Mazarakis N, et al. Examination of novel immunomodulatory effects of L-sulforaphane. Nutrients 13, (2021). 
24. Royce SG, Patel KP, Samuel CS. Characterization of a novel model incorporating airway epithelial damage and related fibrosis to the pathogenesis of asthma. Laboratory Investigation 94, 1326-1339 (2014).

25. Casaro M, Souza VR, Oliveira FA, Ferreira CM. OVA-induced allergic airway inflammation mouse model. In: Pre-Clinical Models: Techniques and Protocols. (ed ${ }^{\wedge}\left(e^{d}\right.$ Guest PC). Springer New York (2019).

26. Kim DI, Song M-K, Lee K. Comparison of asthma phenotypes in OVA-induced mice challenged via inhaled and intranasal routes. BMC Pulmonary Medicine 19, 241 (2019).

27. Bolden JE, Peart MJ, Johnstone RW. Anticancer activities of histone deacetylase inhibitors. Nature Reviews Drug Discovery 5, 769-784 (2006).

28. Trott O, Olson AJ. AutoDock Vina: improving the speed and accuracy of docking with a new scoring function, efficient optimization, and multithreading. J Comput Chem 31, 455-461 (2010).

29. Somoza JR, et al. Structural snapshots of human HDAC8 provide insights into the class I histone deacetylases. Structure 12, 1325-1334 (2004).

30. Mazarakis N, et al. Investigation of molecular mechanisms of experimental compounds in murine models of chronic allergic airways disease using synchrotron Fourier-transform infrared microspectroscopy. Sci Rep 10, 11713 (2020).

31. Wardyn JD, Ponsford AH, Sanderson CM. Dissecting molecular cross-talk between Nrf2 and NF$\kappa \mathrm{B}$ response pathways. Biochemical Society Transactions 43, 621-626 (2015).

32. Sun X, et al. NRF2 preserves genomic integrity by facilitating ATR activation and G2 cell cycle arrest. Nucleic Acids Research 48, (2020).

33. Peng D, et al. NRF2 antioxidant response protects against acidic bile salts-induced oxidative stress and DNA damage in esophageal cells. Cancer Letters 458, 46-55 (2019).

34. Gozzelino R, Jeney V, Soares MP. Mechanisms of cell protection by heme oxygenase-1. Annual Review of Pharmacology and Toxicology 50, 323-354 (2010).

35. Kitada O, et al. Heme oxygenase-1 (HO-1) protein induction in a mouse model of asthma. Clin Exp Allergy 31, 1470-1477 (2001).

36. Al-Harbi NO, et al. Sulforaphane treatment reverses corticosteroid resistance in a mixed granulocytic mouse model of asthma by upregulation of antioxidants and attenuation of Th17 immune responses in the airways. European Journal of Pharmacology 855, 276-284 (2019). 
37. Dokmanovic M, Clarke C, Marks PA. Histone deacetylase inhibitors: overview and perspectives. Molecular Cancer Research 5, 981 (2007).

38. Starrett W, Blake DJ. Sulforaphane inhibits de novo synthesis of IL-8 and MCP-1 in human epithelial cells generated by cigarette smoke extract. Journal of Immunotoxicology 8, 150-158 (2011).

39. Elliot JG, Donovan GM, Wang KCW, Green FHY, James AL, Noble PB. Fatty airways: implications for obstructive disease. European Respiratory Journal 54, 1900857 (2019).

40. Higami Y, et al. Increased epicardial adipose tissue is associated with the airway dominant phenotype of chronic obstructive pulmonary disease. PLos one 11, e0148794 (2016).

41. Adcock IM, Tsaprouni L, Bhavsar P, Ito K. Epigenetic regulation of airway inflammation. Curr Opin Immunol 19, 694-700 (2007).

42. Royce SG, Karagiannis TC. Histone deacetylases and their role in asthma. J Asthma 49, 121-128 (2012).

43. Ito K, et al. Expression and activity of histone deacetylases in human asthmatic airways. Am J Respir Crit Care Med 166, 392-396 (2002).

44. Khurana I, et al. SAHA attenuates Takotsubo-like myocardial injury by targeting an epigenetic Ac/Dc axis. Signal Transduction and Targeted Therapy 6, 159 (2021).

45. Barnes PJ. Mechanisms and resistance in glucocorticoid control of inflammation. J Steroid Biochem Mol Biol 120, 76-85 (2010).

46. Adcock IM. HDAC inhibitors as anti-inflammatory agents. Br J Pharmacol 150, 829-831 (2007).

47. Xu WS, Parmigiani RB, Marks PA. Histone deacetylase inhibitors: molecular mechanisms of action. Oncogene 26, 5541-5552 (2007).

48. Karagiannis TC, El-Osta A. Will broad-spectrum histone deacetylase inhibitors be superseded by more specific compounds? Leukemia 21, 61-65 (2007).

49. Lunke S, et al. Epigenetic evidence of an Ac/Dc axis by VPA and SAHA. Clinical Epigenetics 13, 58 (2021).

50. Pirola L, et al. Genome-wide analysis distinguishes hyperglycemia regulated epigenetic signatures of primary vascular cells. Genome Research 21, 1601-1615 (2011). 
51. Rafehi H, et al. Vascular histone deacetylation by pharmacological HDAC inhibition. Genome $3 \quad \operatorname{Res} 24,1271-1284$ (2014).

4

52. Royce SG, et al. Protective effects of valproic acid against airway hyperresponsiveness and airway remodeling in a mouse model of allergic airways disease. Epigenetics 6, 1463-1470 (2011).

53. Royce SG, et al. Effects of the histone deacetylase inhibitor, trichostatin A, in a chronic allergic airways disease model in mice. Arch Immunol Ther Exp (Warsz) 60, 295-306 (2012).

54. Choi JH, Oh SW, Kang MS, Kwon HJ, Oh GT, Kim DY. Trichostatin A attenuates airway inflammation in mouse asthma model. Clin Exp Allergy 35, 89-96 (2005).

55. Ren Y, et al. Therapeutic effects of histone deacetylase inhibitors in a murine asthma model. Inflamm Res 65, 995-1008 (2016).

56. Ho E, Clarke JD, Dashwood RH. Dietary sulforaphane, a histone deacetylase inhibitor for cancer prevention. J Nutr 139, 2393-2396 (2009).

57. Myzak MC, Tong P, Dashwood WM, Dashwood RH, Ho E. Sulforaphane retards the growth of human PC-3 xenografts and inhibits HDAC activity in human subjects. Exp Biol Med (Maywood) 232, 227-234 (2007).

58. Myzak MC, Ho E, Dashwood RH. Dietary agents as histone deacetylase inhibitors. Mol Carcinog 45, 443-446 (2006).

59. Li ML, Su XM, Ren Y, Zhao X, Kong LF, Kang J. HDAC8 inhibitor attenuates airway responses to antigen stimulus through synchronously suppressing galectin-3 expression and reducing macrophage-2 polarization. Respir Res 21, 62 (2020).

60. Hubbert C, et al. HDAC6 is a microtubule-associated deacetylase. Nature 417, 455-458 (2002).

61. Nian H, Delage B, Ho E, Dashwood RH. Modulation of histone deacetylase activity by dietary isothiocyanates and allyl sulfides: studies with sulforaphane and garlic organosulfur compounds. Environ Mol Mutagen 50, 213-221 (2009).

62. Dashwood RH, Myzak MC, Ho E. Dietary HDAC inhibitors: time to rethink weak ligands in cancer chemoprevention? Carcinogenesis 27, 344-349 (2006).

63. Myzak MC, Dashwood RH. Histone deacetylases as targets for dietary cancer preventive agents: lessons learned with butyrate, diallyl disulfide, and sulforaphane. Curr Drug Targets 7, 443-452 (2006). 
64. Wu W, Peng G, Yang F, Zhang Y, Mu Z, Han X. Sulforaphane has a therapeutic effect in an atopic dermatitis murine model and activates the Nrf2/HO1 axis. Mol Med Rep 20, 1761-1771 (2019).

65. Yan B, et al. Sulforaphane prevents bleomycin-induced pulmonary fibrosis in mice by inhibiting oxidative stress via nuclear factor erythroid 2-related factor-2 activation. Mol Med Rep 15, 40054014 (2017).

66. Melgert BN, et al. Female mice are more susceptible to the development of allergic airway inflammation than male mice. Clin Exp Allergy 35, 1496-1503 (2005).

67. Hayashi T, Adachi Y, Hasegawa K, Morimoto M. Less sensitivity for late airway inflammation in males than females in BALB/c mice. Scand J Immunol 57, 562-567 (2003).

68. Royce SG, Miao YR, Lee M, Samuel CS, Tregear GW, Tang ML. Relaxin reverses airway remodeling and airway dysfunction in allergic airways disease. Endocrinology 150, 2692-2699 (2009).

69. Donovan C, et al. Differential effects of allergen challenge on large and small airway reactivity in mice. PLoS One 8, e74101 (2013).

70. Donovan C, Royce SG, Vlahos R, Bourke JE. Lipopolysaccharide does not alter small airway reactivity in mouse lung slices. PLoS One 10, e0122069 (2015).

71. Bray NL, Pimentel H, Melsted P, Pachter L. Near-optimal probabilistic RNA-seq quantification. Nat Biotechnol 34, 525-527 (2016).

72. Love MI, Huber W, Anders S. Moderated estimation of fold change and dispersion for RNA-seq data with DESeq2. Genome Biol 15, 550 (2014).

73. Mootha VK, et al. PGC-1alpha-responsive genes involved in oxidative phosphorylation are coordinately downregulated in human diabetes. Nat Genet 34, 267-273 (2003).

74. Kaspi A, Ziemann M. mitch: multi-contrast pathway enrichment for multi-omics and single-cell profiling data. BMC Genomics 21, 447 (2020).

75. Wickham HD. ggplot2: Elegant Graphics for Data Analysis. Springer-Verlag (2016).

76. Ververis K, Karagiannis TC. An atlas of histone deacetylase expression in breast cancer: fluorescence methodology for comparative semi-quantitative analysis. Am J Transl Res 4, 24-43 (2012). 
1 77. Bressi JC, et al. Exploration of the HDAC2 foot pocket: Synthesis and SAR of substituted N-(2-

2 aminophenyl)benzamides. Bioorganic \& Medicinal Chemistry Letters 20, 3142-3145 (2010).

3

4 78. Watson PJ, Fairall L, Santos GM, Schwabe JWR. Structure of HDAC3 bound to co-repressor and $5 \quad$ inositol tetraphosphate. Nature 481, 335-340 (2012).

6

79. Lindahl A, Hess, \& van der Spoel. GROMACS 2020.4 Manual. (ed^(eds Zenodo.) (2020).

80. Dallakyan S. OAJ. Small-molecule library screening by docking with PyRx. Humana Press 10

11

12

13

14

15

16

17

18

19

20

21

22

23

24

25 


\section{Figure Legends}

\section{Figure 1. L-sulforaphane protects from bronchial mucosal damage in a murine chronic} sulforaphane (LSF), and broad-spectrum histone deacetylase inhibitor suberoylanilide hydroxamic acid (SAHA) (A), used in this study. The ovalbumin (OVA)-induced chronic murine model of allergic airway disease (AAD) was investigated (B). Hematoxylin and eosin (H\&E) stained sections of formalin-fixed paraffin-embedded mouse lung tissue derived from the

8 prevention model show LSF attenuates epithelial thickening induced by OVA. Little or no

9 peribronchial inflammatory infiltrate was observed for the saline and OVA-LSF mice, while severe to moderate peribronchial inflammatory infiltrate was present in the OVA-vehicle (OVA-

$11 \mathrm{VEH}$ ) and OVA-SAHA mice respectively (C). Immunofluorescence staining of Annexin V (red),

12 a membrane apoptotic marker and histone deacetylase enzyme (HDAC) 8 (green) (D). Strong

13 staining of Annexin V was found in the bronchial epithelium and peribronchial inflammatory 14 cells in mice treated with OVA-VEH and OVA-SAHA. Weak Annexin V staining was observed

15 in the OVA-LSF mice and no staining was found in the epithelium of the control saline group.

16 Merged image: nucleus stained with 4',6-diamidino-2-phenylindole (DAPI) (blue), Annexin V

17 (red), HDAC8 (green). Morphometric analysis of epithelial thickness (E) and subepithelial

18 collagen (F) from H\&E stained lung sections shows both OVA-VEH and OVA-SAHA mice significantly elevated epithelial and subepithelial thickness compared to saline and OVA-LSF

20 mice. Image $\mathrm{J}$ analysis of the mean fluorescence intensity of Annexin $\mathrm{V}$ expression showed

21 significantly elevated expression in OVA-VEH and OVA-SAHA mice compared to saline and 22 OVA-LSF mice. Data shown as the mean \pm SEM; $* * p<0.01$ and $* * * p<0.001$. 
2 allergic airways disease. In the reversal model, L-sulforaphane (LSF) was administered

3 following ovalbumin (OVA) sensitization (A). Representative photomicrographs of histological

4 stained sections of formalin-fixed paraffin-embedded mouse lung tissue derived from the

5 reversal model (B). Hematoxylin and eosin (H\&E) staining shows LSF reduces peribronchial

6 inflammatory cell infiltrate induced by OVA (Bi). Masson's trichrome staining shows a thin

7 epithelium (arrow) and little subepithelial collagen (arrowhead) in the saline and OVA-LSF mice

8 (Bii). Airways in OVA-vehicle (OVA-VEH) mice display epithelial thickening (arrow) and

9 severe subepithelial collagen deposition (arrowhead). Silver impregnation staining highlights that

LSF attenuates the increased reticular fibers $(\mathrm{R})$ and collagen fibers $(\mathrm{C})$ found in the OVA-VEH

11 mice (Biii). Gomori's aldehyde-fuchsin staining shows an increase of elastic fibers (royal blue)

12 surrounding the inner and outer walls of the smooth muscle in the OVA-VEH mice that is not

13 present in the saline and OVA-LSF mice (Biv). Blood vessels (BV) stained positive. Sirius red

14 staining imaged under polarized light differentiates between collagen type I (red/yellow) and

15 type III (green) in lung sections (Bv). Morphometric analysis of Masson's trichrome stained

16 sections shows OVA-VEH had significantly elevated epithelial thickness (C) and subepithelial

17 collagen thickness (D) when compared to both saline and OVA-LSF mice. Analysis of type I and

18 III collagen from sirius red stained sections indicates that OVA-VEH significantly elevates type I

19 collagen when compared to all other groups (E). Airway hyperresponsiveness to methacholine

20 was recorded using plethysmography 48 hours following the final treatment and nebulization $(\mathrm{F})$.

21 Increasing doses of methacholine was administered and maximal resistance values

$22\left(\mathrm{cmH}_{2} \mathrm{O} / \mathrm{mL} / \mathrm{sec}\right)$ after $5 \mathrm{~min}$ was recorded. OVA-VEH mice had elevated airway resistance at

23 the highest methacholine dose as compared to saline mice. OVA-LSF mice had significantly 
1 lower airway resistance at the highest methacholine dose compared to those of OVA-VEH mice.

2 All data represented as the mean $\pm \mathrm{SEM} ; *^{*}<0.05 * * p<0.01$ and $* * * p<0.001$.

4 Figure 3. Molecular effects of L-sulforaphane in the chronic allergic airways disease model.

5 Immunohistochemistry was performed on formalin-fixed paraffin-embedded mouse lung tissue

6 derived from the reversal model. Representative photomicrographs of $\alpha$-smooth muscle actin $(\alpha-$

7 SMA) expression (Ai) and immunohistochemical morphometric analysis (Aii) shows staining for

$8 \alpha$-SMA in the myofibroblasts in the lamina propria was significantly elevated in ovalbumin-

9 vehicle (OVA-VEH) mice compared to saline and ovalbumin-L-sulforaphane (OVA-LSF) mice.

10 LSF significantly attenuated the reduced expression of Caveolin-1 following OVA-sensitization

11 in OVA-VEH mice as observed by immunohistochemical images of Caveolin-1 (Bi) and

12 morphometric analysis in bronchial epithelium (Bii). Data shown as the mean $\pm \mathrm{SEM} ;{ }^{*} p<0.05$

13 and $* * * p<0.001$. The comparison of small airway responses to methacholine (MCh) in precision

14 cut lung slices from OVA-VEH mice (C). Representative traces show bronchial constriction as a

15 percent of the initial lumen area in response to $300 \mathrm{nM} \mathrm{MCh}$ and subsequent bronchial dilation

16 following a step-wise increase in concentrations of LSF to a maximum of $15 \mu \mathrm{M}(\mathrm{Ci})$ and $30 \mu \mathrm{M}$

17 (Cii). Focal plane array-Fourier-transform infrared spectroscopy (FPA-FTIR) chemical images of

18 lipid distributions of lung sections derived from the reversal model (Di). Average absorbance

19 spectra (obtained from >100 individual spectra) from the bronchial epithelium (Dii) and their

20 corresponding second derivative spectra (Diii) highlight key changes in spectral patterns in lipid

21 regions.

22 
Figure 4. L-sulforaphane modulates key antioxidant defense pathways in both the

\section{prevention and reversal model of chronic allergic airways disease. Representative} immunofluorescent photomicrographs of lung sections stained with nuclear factor erythroid 2related factor 2 (Nrf2) (red) show strong staining in ovalbumin-L-sulforaphane (OVA-LSF) mice in both the prevention and reversal model (A). Merged image: nucleus stained with 4',6diamidino-2-phenylindole (DAPI) (blue), Nrf2 (red). Nrf2 was analyzed using Image J for mean fluorescence (FL) intensity (Bi, iii), number of focal points (Bii, iv) and co-localization to DAPI staining (C). Nrf2 expression was significantly elevated in both the prevention (Bi) and reversal (Biii) models, and the number of foci also significantly increased in the prevention (Bii) and reversal (Biv) models. Line scan analysis indicated that Nrf2 expression colocalizes predominantly with heterochromatin according to DAPI staining in both saline and OVA-vehicle (OVA-VEH) mice lung sections. Nrf2 was sometimes found outside heterochromatic regions in OVA-LSF mouse lungs (C). Immunohistochemical images of superoxide dismutase 2 (SOD2) in paraffin-embedded mouse lung tissue derived from the reversal model (Di). Morphometric analysis showed a significant elevation of SOD2 protein expression in the bronchial epithelium in OVA-LSF mice compared to OVA-VEH mice (Dii). Immunofluorescence photomicrographs of lung sections stained with heme oxygenase-1 (HO-1) (red) and DAPI (blue) in the reversal model of allergic airways disease (AAD) (Ei). Analysis of the mean FL intensity of HO-1 showed significant elevation of HO-1 expression in the OVAVEH mice in both the prevention (Eii) and reversal (Eiii) models. OVA-LSF treatment significantly decreased the HO-1 elevation in both models. All data represented as the mean \pm $\mathrm{SEM} ; * p<0.05 * * p<0.01$ and $* * * p<0.001$ 

genes. Ovalbumin-L-sulforaphane (OVA-LSF) significantly attenuates inflammation observed in the ovalbumin-vehicle (OVA-VEH) mice in both the prevention and reversal model

4 (A). Inflammatory cell infiltration of hematoxylin and eosin stained sections of mouse airways was scored by two independent blinded investigators using the scale: $0=$ no inflammation; $1=3$

6 layers of inflammatory cells; $2=6$ layers; $3=10$ layers and congestion; or $4=>10$ layers of

7 inflammatory cells and severe congestion. Representative immunohistochemical

8 photomicrographs detecting phosphorylated Smad2 (Bi) performed on formalin-fixed paraffin-

9 embedded mouse lung tissue derived from the reversal model. Morphometric analysis of stained sections shows OVA-LSF mice had a significant increase of phosphorylated

11 Smad2 (Bii) expression compared to the saline and OVA-VEH mice. The anti-inflammatory

12 effect of LSF was explored in peripheral blood mononuclear cells (PMBC) from healthy adults

$13(n=4)$. PBMCs were treated with the indicated concentrations of LSF and $10 \mu \mathrm{M}$ of

14 suberoylanilide hydroxamic acid (SAHA) (C), the chromatin modifying control compound. An

15 array of chemokines and cytokines were measured by the multiplex bead array assay as

16 indicated. All data is represented as the mean \pm SEM; $* p<0.05 * * p<0.01$ and $* * * p<0.001$. RNA-

17 Seq was performed on adult PBMCs cultured with or without $15 \mu \mathrm{M}$ LSF for 24 hours.

18 Differentially expressed (DE) genes were subjected to gene set enrichment analysis (GSEA) of 19 gene ontology (GO) gene sets and examined for effect (D). Heat map of the top 50 gene sets

20 according to effect demonstrates predominate downregulation of sets related to immune response

21 (Di). The sets as shown are ordered by GSEA enrichment score according to analysis of

22 combined DE genes $\mathrm{n}=4$ (first column); individual sample data is also shown (columns 2 to 5).

23 Heat map color gradient indicates GSEA enrichment score. Differential expression of genes 
1 from selected gene sets (Dii). Genes from the top 100 gene sets ranked by effect were filtered for

2 key terms including chemokine, cytokine, immune and inflammation. Individual genes (FDR <

$3 \quad 0.05)$ are shown with $\log 2$ fold change alongside their gene set and corresponding GSEA

4 enrichment score.

6 Figure 6. L-sulforaphane as a potential selective histone deacetylase 8 inhibitor. Lung

7 sections stained with anti-acetylated histone (red) and anti-acetylated $\alpha$-tubulin (green) show

8 strong staining in ovalbumin-L-sulforaphane (OVA-LSF) mice (A). Merged image: nucleus

9 stained with DAPI (blue), acetylated histones H3 or H2B (red), acetylated $\alpha$-tubulin (green).

H2Bac was analyzed in both models (B); H3ac and acetylated $\alpha$-tubulin analyzed in the

11 prevention model (C). Histone deacetylase (HDAC) 8 expression investigated in the prevention

12 model of chronic allergic airways disease (AAD) (D). LSF reduces the enzymatic activity of

$13 \mathrm{HDAC} 8 ; \mathrm{EC}_{50}=92.07 \pm 7.45 \mu \mathrm{M}$ (SAHA $\mathrm{EC}_{50}=306 \mathrm{nM}$ in the same assay) (E). Data

14 represented as the mean \pm SEM; ${ }^{*} p<0.05 * * p<0.01$ and ${ }^{* * *} p<0.001$. Western blot shows $15 \mu \mathrm{M}$

15 LSF decreases HDAC8 expression and increases acetylated $\alpha$-tubulin and H3ac in A549 lung

16 cells (positive control $=10 \mu \mathrm{M}$ SAHA) $(\mathrm{F})$. Molecular docking of LSF to HDAC8 (G). Aligned

17 structures of HDAC8, for PDB ID: 1 T64 (1 pocket, green); 1T67 (2 pockets, red) and 1VKG (1

18 groove, purple). Black sphere represents $\mathrm{Zn}$. Viewed along axis parallel (Gi) and perpendicular

19 to helix bundle (Gii). LSF binding sites to HDAC8, 1 pocket (green), 2 pockets (purple) and 1

20 groove (red). Helix-loop B-helix motif highlighted in black. Viewed along axis parallel (Giii)

21 and perpendicular to helix bundle (Giv). Predicted binding pose of LSF to the tertiary-deep sub-

22 site of 1 pocket of HDAC8. Residues 21-49 are shown in ribbon representation. HDAC

23 sidechains in contact with LSF are shown in bonds representation (Gv). The tertiary-deep sub- 
1 site (green) is predicted for all three HDAC8 structures. The tertiary-interm sub-site (purple) is

2 predicted for the 1 pocket and 2 pockets structures. The tertiary-shallow sub-site (red) is only

3 predicted for the 1 groove structure. Residues 21-49 are shown in opaque grey ribbon

4 representation (Gvi). Potential steric clash between the residue 21-31 (HDAC8 numbering) helix

5 region of HDAC3 (grey ribbon) and LSF (green spheres). The corresponding helix HDAC8 (red

6 ribbon) is shown for comparison. Sequence alignment between HDAC3 and HDAC8 shows the

7 extra two residues present for the former isoform compared to the latter are partially responsible

8 for occlusion of the tertiary site (Gvii).

9

Figure 7. Mechanisms associated with the attenuation of ovalbumin-induced allergic

11 airways disease by L-sulforaphane. Summary of the changes that occur to normal bronchioles

12 in the ovalbumin (OVA)-induced chronic murine model of allergic airways disease (AAD) as

13 observed in this study. The mechanisms associated with prevention and reversal of the

14 pathobiology by L-sulforaphane (LSF) are depicted; antioxidant, anti-inflammatory and

15 epigenetic mechanisms of action are shown. Methodologies used to detect changes are in

16 indicated in brackets; FL = immunofluorescence.

17

18

19

20

21

22 
A<smiles>C[S@](=O)CCCCN=C=S</smiles>

L-sulforaphane (LSF)<smiles>O=C(CCCCCCC(=O)Nc1ccccc1)NO</smiles>

Vorinostat (SAHA)

\section{B Prevention Model}

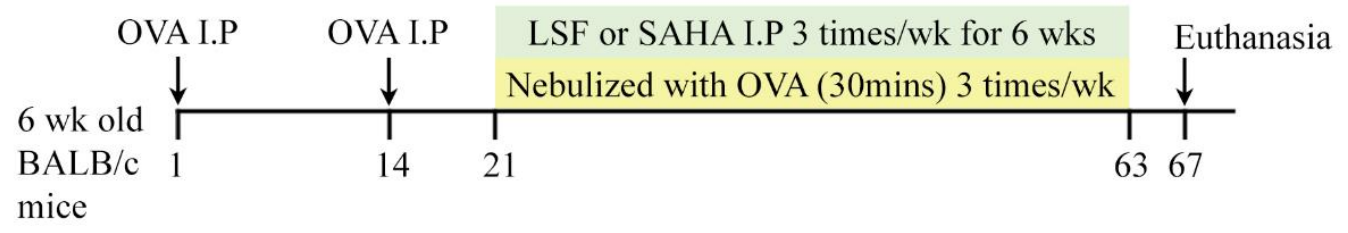

C

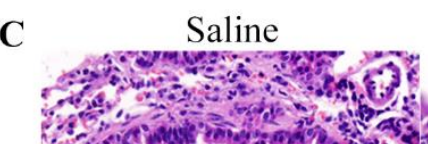

OVA-VEH

OVA-SAHA

OVA-LSF

$\stackrel{11}{1}$
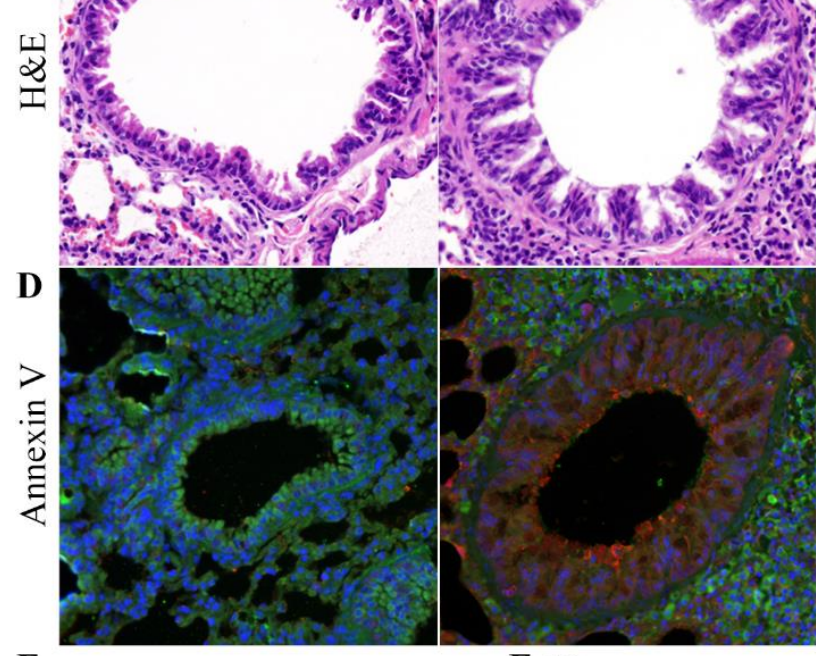

E

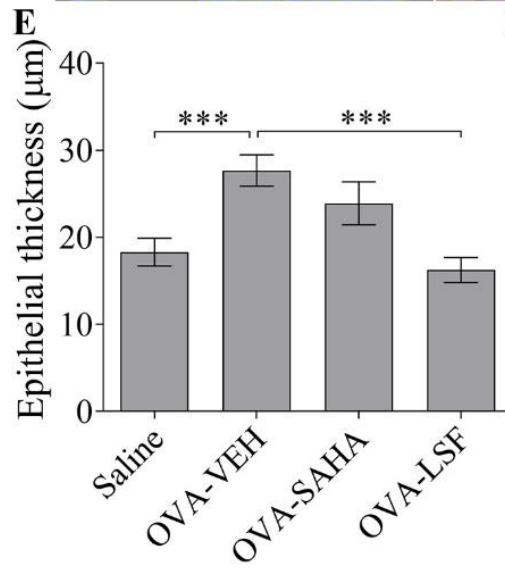

F

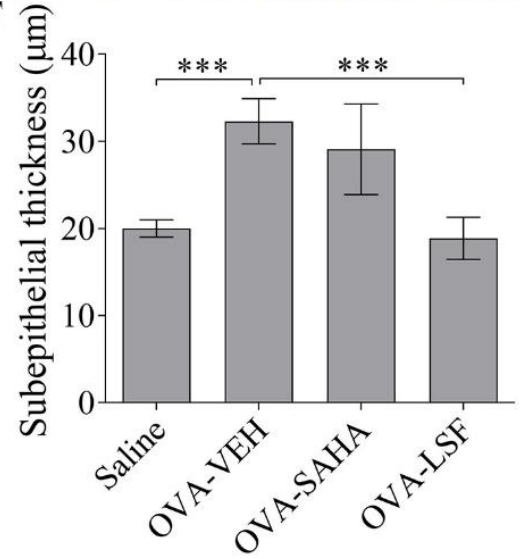

$200 \mu \mathrm{m}$

G

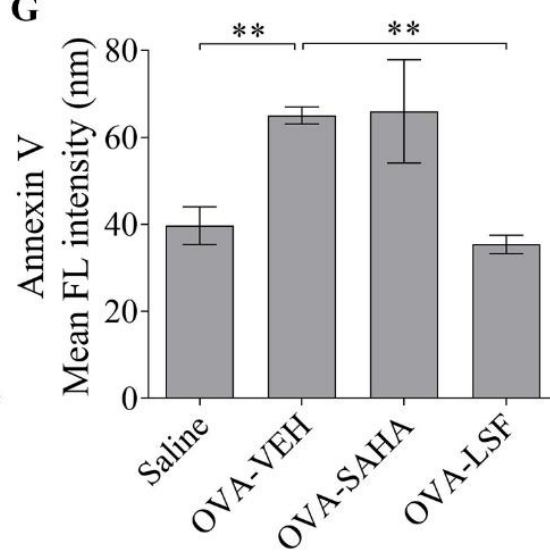


2 allergic airways disease.

A
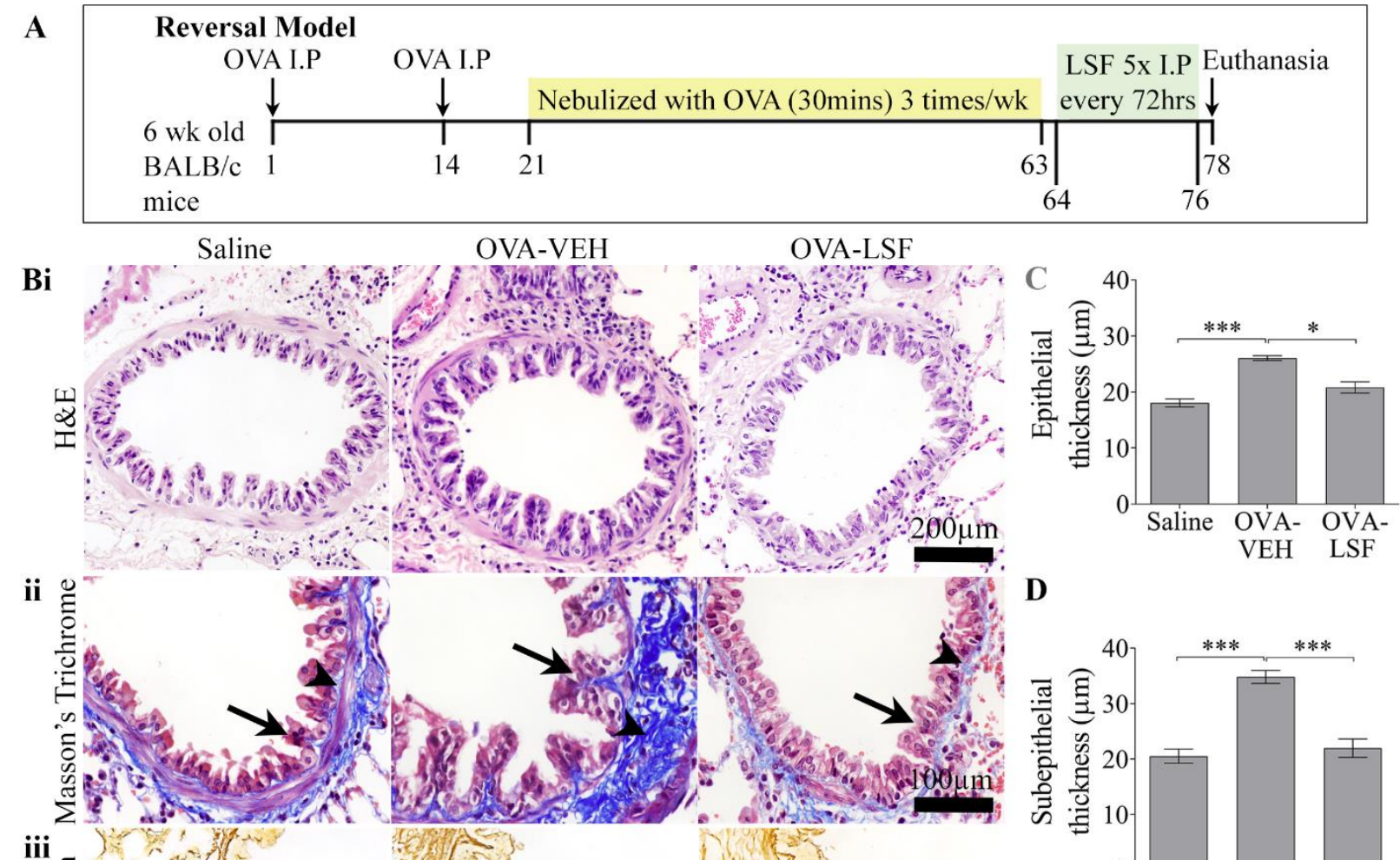

D
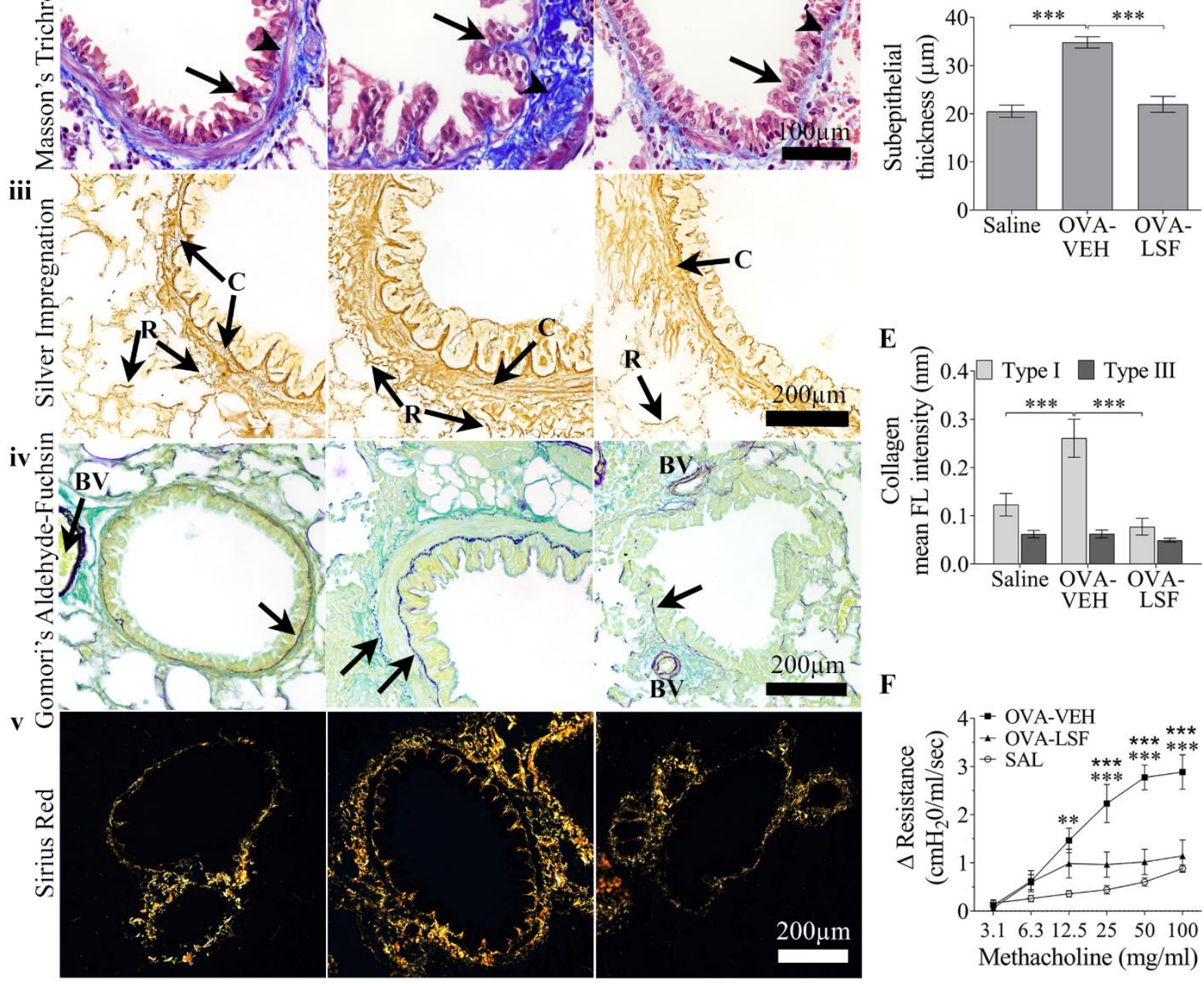

F

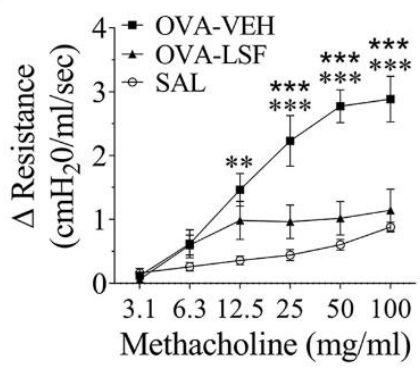


Figure 3. Molecular effects of L-sulforaphane in the chronic allergic airways disease model.
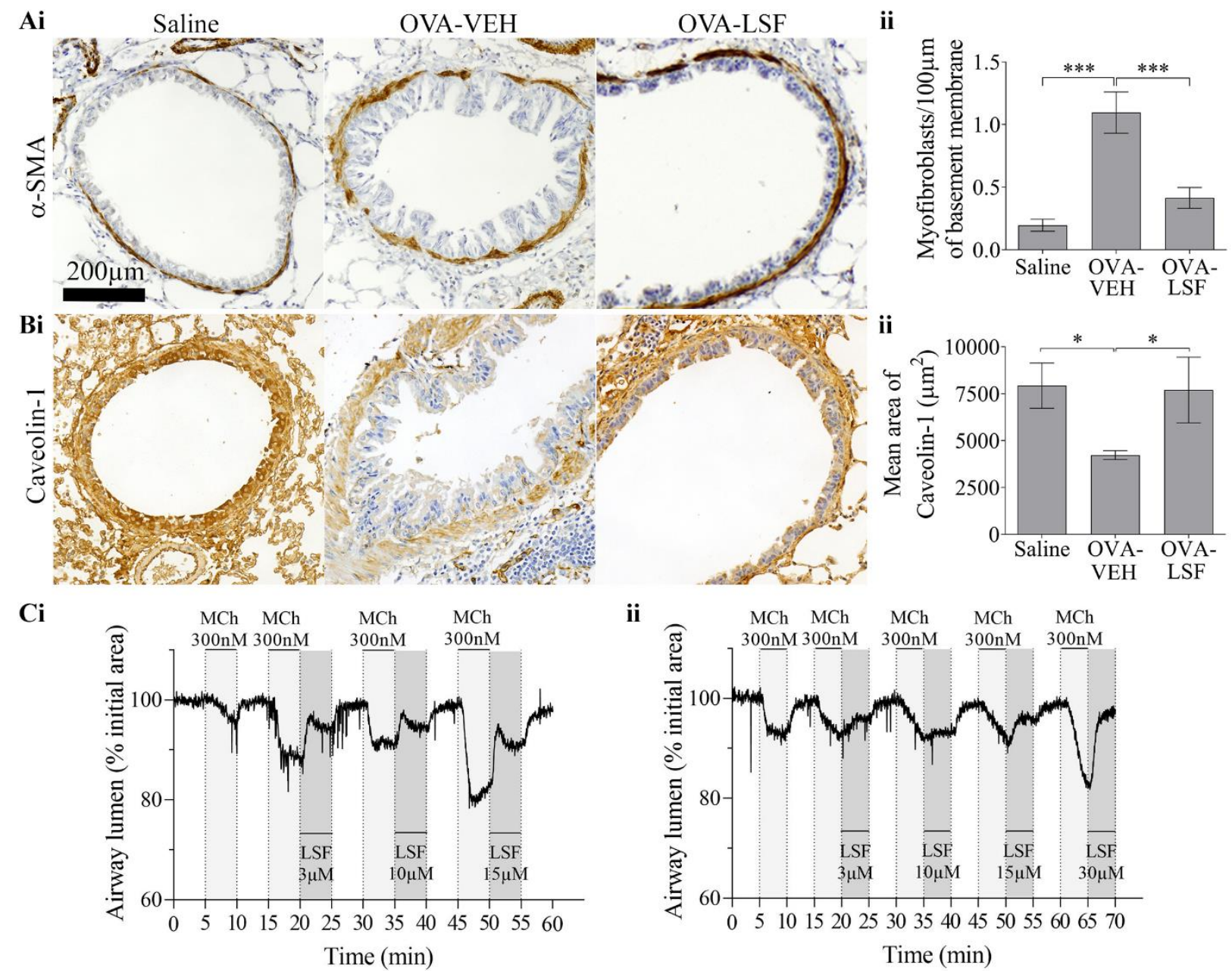

ii
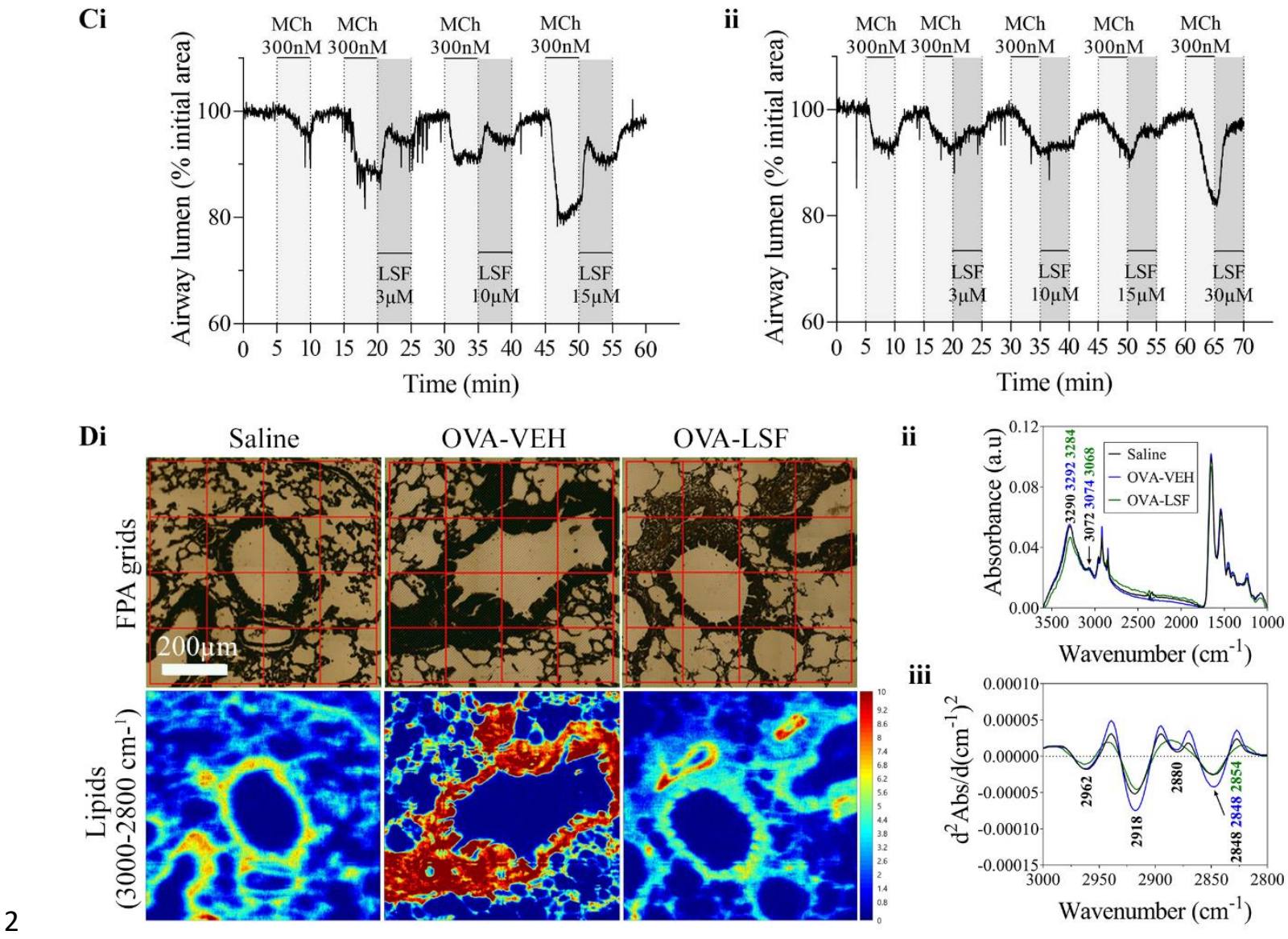
Figure 4. L-sulforaphane modulates key antioxidant defense pathways in both the

2 prevention and reversal model of chronic allergic airways disease.

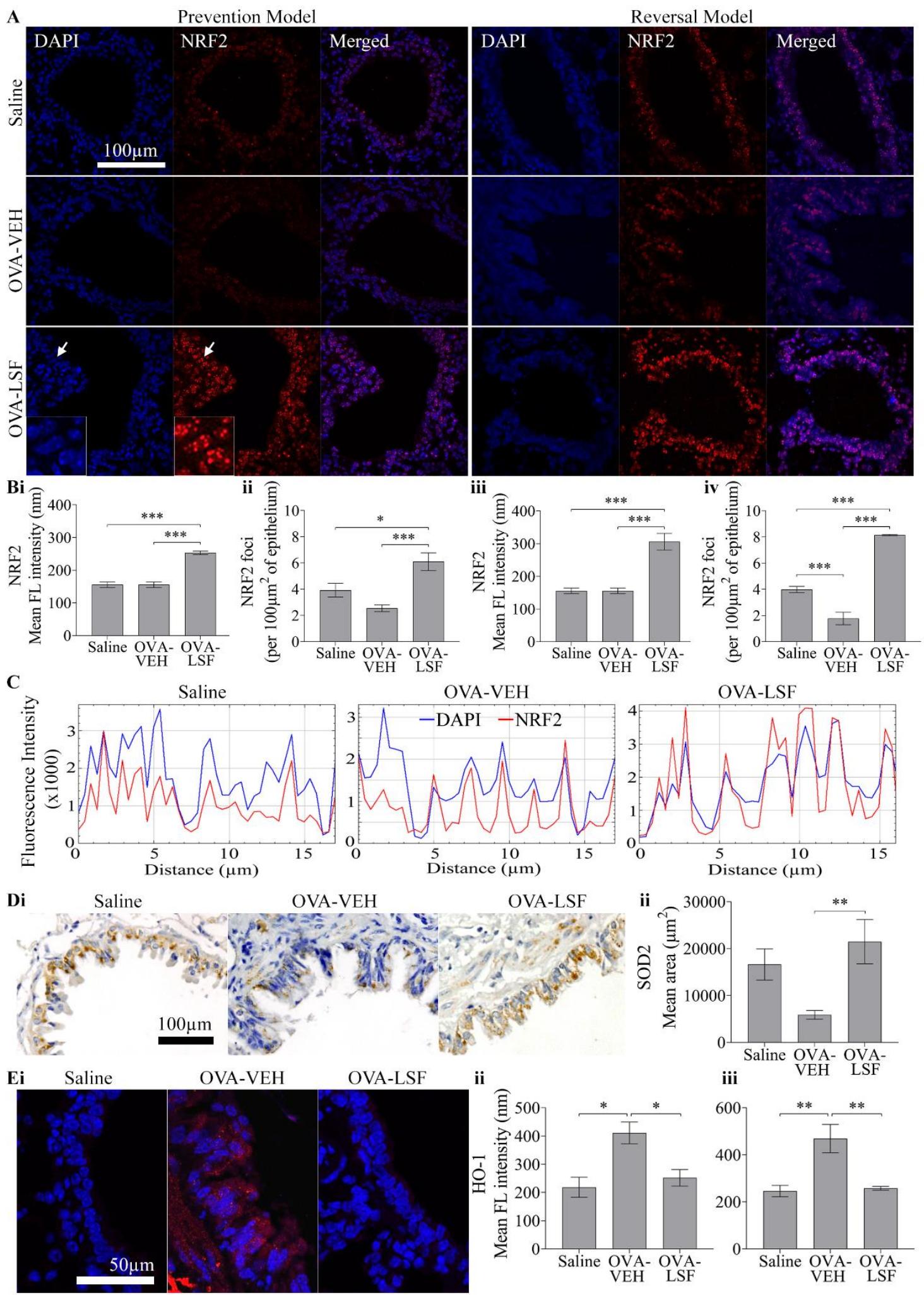



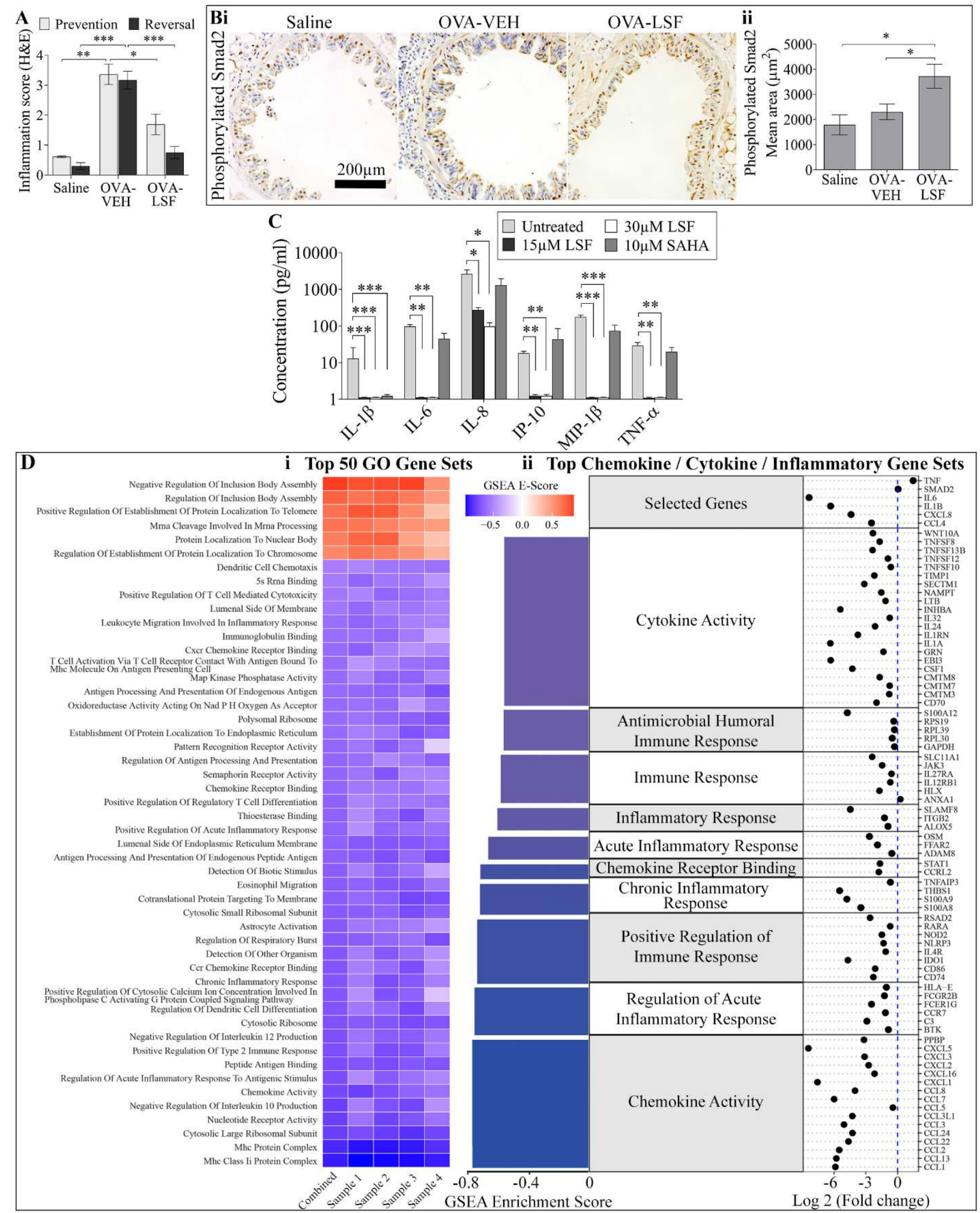
Figure 6. L-sulforaphane as a potential selective histone deacetylase 8 inhibitor.
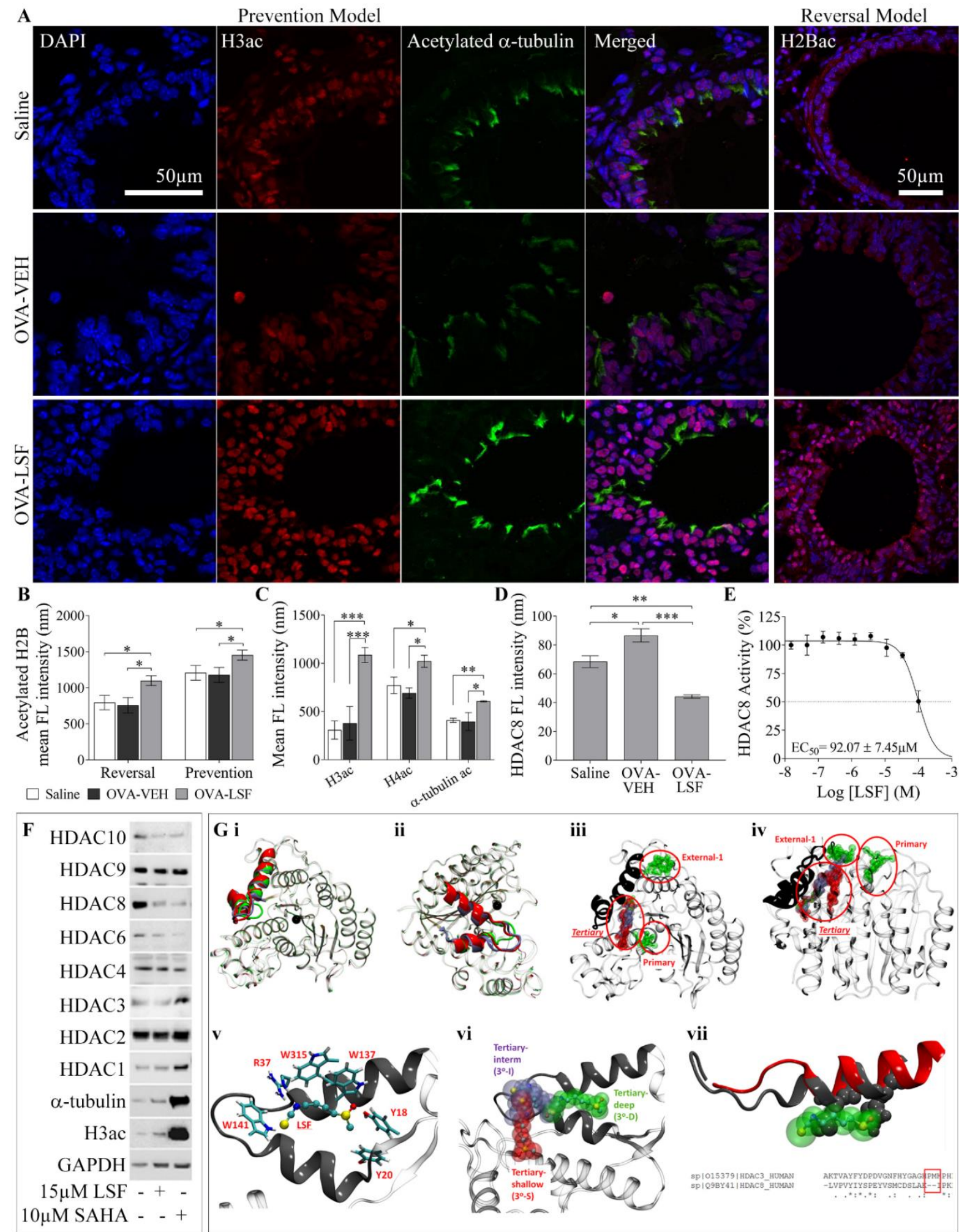

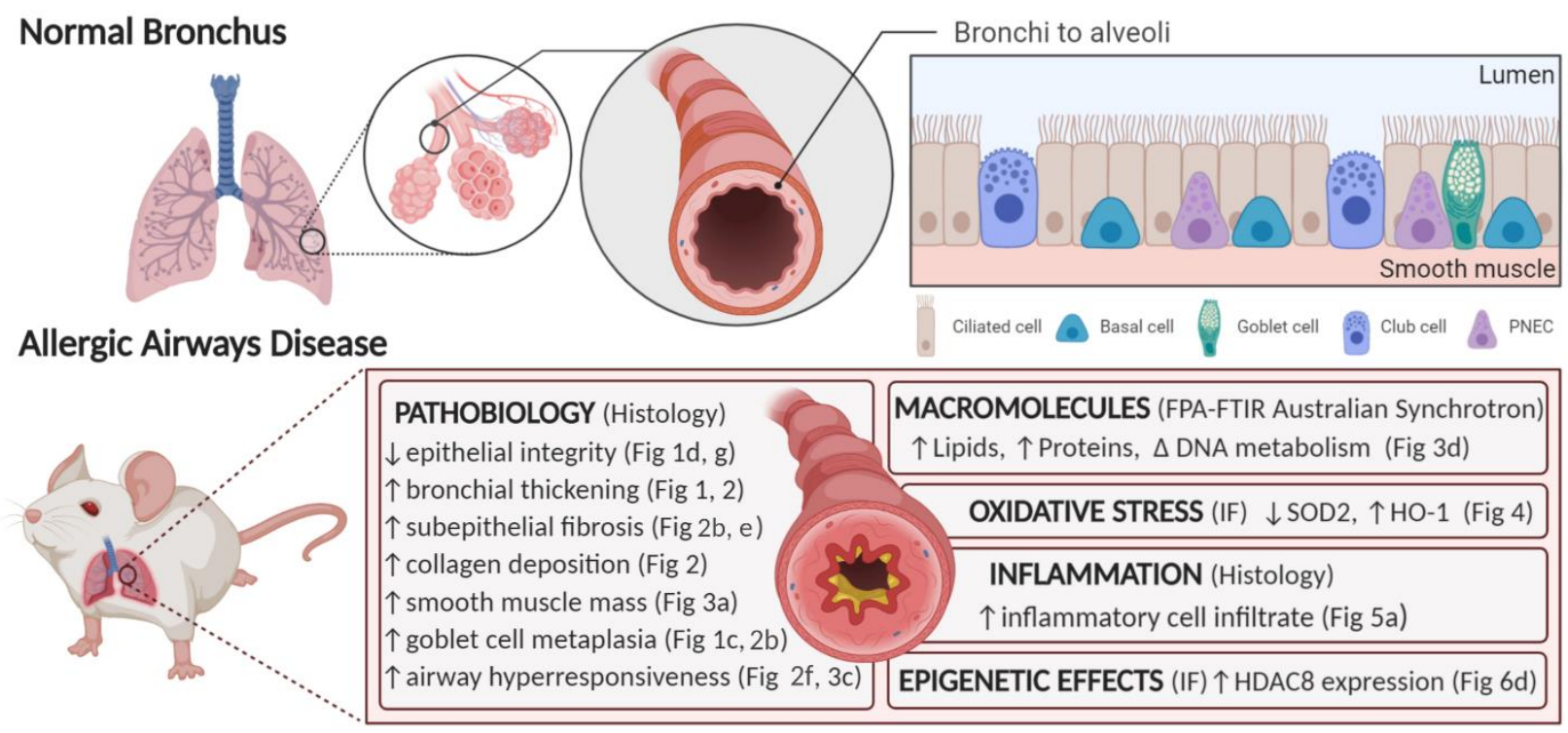

\section{Effects of L-sulforaphane in Allergic Airways Disease}

\begin{tabular}{|c|c|c|}
\hline \multirow{6}{*}{$\begin{array}{l}\text { PATHOBIOLOGY (Histology) } \\
\uparrow \text { epithelial integrity (Fig 1d, g) } \\
\downarrow \text { bronchial thickening (Fig 1, 2) } \\
\downarrow \text { subepithelial fibrosis (Fig 2b, e) } \\
\downarrow \text { collagen deposition (Fig 2) } \\
\downarrow \text { smooth muscle mass (Fig 3a) } \\
\downarrow \text { goblet cell metaplasia (Fig 1c, 2b) } \\
\downarrow \text { airway hyperresponsiveness (Fig 2f, 3c) }\end{array}$} & $\begin{array}{l}\text { MACROMOLECULES (FPA-FTIR Australian Synchrotron) } \\
\downarrow \text { Lipids, } \downarrow \text { Proteins, } \triangle \text { DNA metabolism (Fig 3d) }\end{array}$ & \multirow{6}{*}{$\begin{array}{c}\text { EPIGENETIC EFFECTS } \\
\text { (Direct Ligand Binding, } \\
\text { IF, Immunoblot, } \\
\text { Molecular Modelling) } \\
\uparrow \text { H2Bac, H3ac, H4ac } \\
\uparrow \text { acetylated a-tubulin } \\
\downarrow \text { HDAC8 expression } \\
\downarrow \text { HDAC8 activity (Fig 6 }\end{array}$} \\
\hline & OXIDATIVE STRESS (IF) $\uparrow$ NRF2, $\uparrow$ SOD2, $\downarrow$ HO-1 (Fig 4) & \\
\hline & INFLAMMATION (Histology, Multiplex Assay, RNA-seq) & \\
\hline & $\downarrow$ inflammatory cell infiltrate (Fig 5a), $\uparrow$ smad2 (Fig 5b) & \\
\hline & $\downarrow$ IL-1 $\beta$, IL-6, IL-8, IP-10, MIP-1 $\beta$, TNFa (Fig 5c) & \\
\hline & $\downarrow$ genes related to immune responses (Fig $5 \mathrm{~d}$ ) & \\
\hline
\end{tabular}


1 Acknowledgments: We acknowledge the intellectual and financial support from McCord

2 Research (Iowa, USA). The authors would like to acknowledge the use of the facilities provided

3 by Monash University (Clayton, VIC, Australia) and the Murdoch Children's Research Institute

4 (Parkville, VIC, Australia) for their care and husbandry of the mice. The Gomori's aldehyde-

5 fuchsin and silver impregnation stains were performed by Ms Laura Leone at the Melbourne

6 University Histology Platform (University of Melbourne, School of Biomedical Sciences,

7 Parkville, VIC, Australia). The authors would like to acknowledge the use of the facilities

8 provided by Monash Micro Imaging (MMI) at the Alfred Research Alliance (ARA, Melbourne,

9 VIC, Australia) and particularly, the expert assistance from Drs Stephen Cody and Iśka

10 Carmichael. FPA-FTIR imaging measurements were undertaken at the IRM beamline at the

11 Australian Synchrotron, part of ANSTO (Clayton, VIC, Australia). Various figures in this

12 manuscript were created with BioRender.com. We thank the National Computing Infrastructure

13 (NCI), and the Pawsey Supercomputing Centre in Australia (funded by the Australian

14 Government). Further, we thank the Spartan High Performance Computing service (University

15 of Melbourne), and the Partnership for Advanced Computing in Europe (PRACE) for awarding

16 the access to Piz Daint, hosted at the Swiss National Supercomputing Centre (CSCS),

17 Switzerland. We acknowledge our use of the gene set enrichment analysis, GSEA software, and

18 Molecular Signature Database (MSigDB) (Subramanian, Tamayo, et al. (2005), PNAS 102,

19 15545-15550, http://www.broad.mit.edu/gsea/).

21 Funding: We would like to acknowledge intellectual and financial support by McCord Research

22 (Iowa, USA). AEO is supported by an National Health and Medical Research Council

23 (NHMRC) Senior Research Fellowship (1154650). PVL is supported by an NHMRC Career 
1 Development Fellowship (1146198). CD is supported by an NHMRC Early Career Postdoctoral

2 Fellowship (1120152). JL is supported by an Australian Government Research Training Program

3 Scholarship.

5 Author contributions: SGR, JEB, MEC, AH, PVL, CSS, KJS, MLKT, AE, and TCK

6 conceptualized the ideas and overarching aims. JEB, MEC, AH, PVL, CSS, KJS, MJT, JV,

7 MLKT, AE, and TCK were involved in supervision. KV, CD, AH, IK, JL, SM, NM, EP, MJT,

$8 \mathrm{JV}$, and MZ participated in the development and design of the methodology. KV, CD, IK, SM,

$9 \mathrm{NM}, \mathrm{MJT}, \mathrm{JV}$, and MZ conducted the research and investigation process. RCB, AH, JL, EP, and

$10 \mathrm{MZ}$ were responsible for visualization and KV, EP, SGR, PVL, and TCK were involved in

11 production of the first draft of the manuscript.

12

13 Competing interests: Epigenomic Medicine Program (TCK) was supported financially by

14 McCord Research (Iowa, USA), which has a financial interest in dietary compounds including

15 sulforaphane.

16 The remaining co-authors declare that they have no direct financial relation with the commercial

17 identities mentioned in this manuscript that might lead to a conflict of interest.

19 Data and materials availability: RNA sequencing data is available from GEO under the

20 accession GSE160353. To review GEO accession GSE160353 while it remains in private status,

21 go the following address and enter the access token: otmlcuoillwhjcd

22 https://www.ncbi.nlm.nih.gov/geo/query/acc.cgi?acc=GSE160353 